Jannis Burk, Constantin Meyer, Jan Christoph Peters, Sebastian Rauch*

\title{
GIS-gestützte Modellierung der Erreichbarkeiten ambulanter Pflegedienste nach SGB V und SGB XI in Unterfranken unter Berücksichtigung demographischer Entwicklungen
}

\section{GIS-supported modelling of the accessibility of outpatient care services according to SGB V and SGB XI in Lower Franconia under consideration of demographic developments}

https://doi.org/10.2478/rara-2020-0022

Eingegangen: 12. März 2020; Angenommen: 2. Juli 2020

Kurzfassung: Ziel des Beitrags ist die Untersuchung und Bewertung der Erreichbarkeit der Bevölkerung durch ambulante Pflegedienste in Unterfranken unter Einbeziehung der räumlich differenzierten Bevölkerungsstruktur für das Jahr 2017 sowie der voraussichtlichen demographischen Entwicklung bis 2025. Die Erreichbarkeiten werden sowohl rasterbasiert als auch vektorbasiert in einer GIS-Software modelliert. Darüber hinaus werden demographische Entwicklungen in der Altersgruppe „80 Jahre und älter" eingebunden. Die Ergebnisse der rasterbasierten Fahrzeitenmodellierung zeigen flächenhaft relativ gute Erreichbarkeiten, mit Ausnahme weniger peripherer Gebiete. Mittels vektorbasierter Fahrzeitenmodellierung werden Einzugsgebiete, welche auf Personalkosten und Anfahrtspauschalen nach SGB V und SGB XI als Berechnungsgrundlagen basieren, für ambulante Pflegedienste berechnet. Es zeigt sich, dass in Teilen Unterfrankens keine ausreichende Kostendeckung gegeben ist. Hinsichtlich der skizzierten Bevölkerungsentwicklung sowie unzureichender Fahrtkostendeckung im Bereich der Mittelgebirge und abseits der Verdichtungsräume wird eine Flexibilisierung der Anfahrtsvergütung sowie eine Aufstockung des Pflegepersonals empfohlen.

\footnotetext{
*Corresponding author: Sebastian Rauch, Julius-Maximilians-Universität Würzburg, Institut für Geographie und Geologie, Professur für Sozialgeographie, Am Hubland, 97074 Würzburg, Deutschland, E-Mail: sebastian.rauch@uni-wuerzburg.de Jannis Burk, Julius-Maximilians-Universität Würzburg, Institut für Geographie und Geologie, Professur für Sozialgeographie, Am Hubland, 97074 Würzburg, Deutschland

Constantin Meyer, Julius-Maximilians-Universität Würzburg, Institut für Geographie und Geologie, Lehrstuhl für Geographie und Regionalforschung, Am Hubland, 97074 Würzburg, Deutschland Jan Christoph Peters, Julius-Maximilians-Universität Würzburg, Institut für Geographie und Geologie, Professur für Sozialgeographie, Am Hubland, 97074 Würzburg, Deutschland
} 
Abstract: The aim of this paper is to analyse the accessibility of outpatient care services in Lower Franconia, Germany, taking into account the spatially differentiated population structure for the year 2017 as well as the expected demographic development until 2025. The accessibility was modelled both raster-based and vector-based in a GIS software. Moreover, demographic developments in the age group "80 years and older" are also incorporated. The results of the raster-based travel time modelling show relatively good accessibility in the study area except from a few peripheral areas. Catchment areas for outpatient care services were calculated using vector-based travel time modelling, drawing on travel flat rates according to SGB V and SGB XI as well as staff costs as calculation bases for threshold values. In parts of Lower Franconia there is no sufficient cost coverage. With regard to the population development outlined above and insufficient coverage of travel costs by flat-rate travel allowances in low mountain ranges and away from urban agglomerations, a transition to a flexible travel compensation for nursing services and an increase in nursing staff is recommended.

Schlüsselwörter: Erreichbarkeitsanalyse, GIS, Gesundheitswesen, Medizinische Geographie, ambulante Pflege, Unterfranken

Keywords: Accessibility analysis, GIS, Healthcare, Medical geography, Outpatient care, Lower Franconia

\section{Einführung und Themenstellung}

Vor dem Hintergrund des voranschreitenden demographischen Wandels in Deutschland wird der politischen sowie gesamtgesellschaftlichen Debatte zum Thema Pflege besonders in den letzten Jahren ein großer Stellenwert beigemessen. Dabei betont der Gesetzgeber in $\S 3$ des Elften Buches des Sozialgesetzbuchs (SGB XI) grundsätzlich den Anspruch, der häuslichen Pflege Vorrang gegenüber einer vollstationären Pflegeversorgung einzuräumen. Der darin ausgedrückte Grundsatz „ambulant vor stationär" spiegelt sich folglich in der Struktur und Finanzierung des Pflegesystems wider und führt zu einer bedeutsamen Rolle der ambulanten Pflege. Räumlich betrachtet sind es gerade ländlich-periphere Räume, welche durch vergleichsweise größere räumliche Distanzen und eine gewisse Pkw-Abhängigkeit gekennzeichnet sind, in denen ambulante Pflegedienste mit ihrem Leistungsspektrum wichtige Dienstleistungen für Pflegebedürftige wahrnehmen (Heit/Köppe/ Kratschke et al. 2014: $141 \mathrm{f}$.).

In den vergangenen Jahren sind immer wieder Untersuchungen veröffentlicht worden, die Fragen regionaler Erreichbarkeiten von Einrichtungen und Dienstleistungen der Daseinsvorsorge mittels GIS-basierten Erreichbarkeitsanalysen behandeln (Neumeier 2014; Rauch/Rauh 2016; Ahlmeyer/Wittowsky 2018; Rauh/ Rauch 2019). Ambulante Pflegedienste stellen bislang allerdings eher selten einen Untersuchungsgegenstand in Veröffentlichungen dar, wobei als Ausnahmen hier die umfangreichen Beiträge von Neumeier $(2015,2016)$ mit deutschlandweitem Bezugsrahmen zu nennen sind.

Konzeptionell betrachtet lässt sich Erreichbarkeit als ein Konstrukt aus zwei Funktionen verstehen, wobei eine die Aktivität oder zu erreichenden Gelegenheiten (Aktivitätsfunktion) beschreibt, die andere hingegen Mühe, Entfernung oder Kosten, um diese zu erreichen (Widerstandsfunktion) (Spiekermann/Wegener 2012: 12). Für GIS-basierte Erreichbarkeitsanalysen unter Berücksichtigung des zugrunde liegenden Straßennetzes (Netzwerkanalyse) werden in der Regel zwei verschiedene methodische Ansätze verwendet: der (traditionelle) vektorielle oder der Rasteransatz (Neumeier 2015: 13 f.). Da diese je nach Fragestellung spezifische Vor- und Nachteile bieten, werden in dieser Untersuchung beide Ansätze verwendet. Die jeweils erzeugten Einzugs- oder Versorgungsbereiche lassen sich außerdem mit räumlich erfassbaren Daten kombinieren, um daraus örtliche Potenziale für bestimmte Fragestellungen abzuleiten. Diese Daten können etwa Einwohnerzahlen oder andere demographische oder sozioökonomische Daten sein (Rauch/Rauh 2016: 441).

Für diesen Beitrag, der sich mit den räumlichen Erreichbarkeitsverhältnissen ambulanter Pflegedienste im Regierungsbezirk Unterfranken befasst, wurden verschiedene Schwerpunkte gesetzt, um die derzeitige ambulante Pflegeinfrastruktur zu analysieren. Diese basieren auf den folgenden Leitfragen:

- Welche Rolle spielen Erreichbarkeiten und Fahrzeitdistanzen in der ambulanten Pflege - besonders unter Berücksichtigung der sogenannten Anfahrtspauschale der Pflegeversicherung? 
- Wie gestalten sich die gesamträumlichen sowie kleinräumig differenzierten Erreichbarkeitsverhältnisse durch ambulante Pflegedienste in Unterfranken?

- Wie viele potenziell pflegebedürftige Menschen befinden sich (aktuell sowie unter Berücksichtigung von Bevölkerungsprognosen) in den modellierten Einzugsbereichen ambulanter Pflegedienste?

- Wie lässt sich dies im Kontext des demographischen Wandels sowie des potenziellen Fachkräftemangels in der Pflege interpretieren?

In der behandelten Untersuchung wurde ein Methodenmix angewandt, welcher neben den vektoriellen und rasterbasierten Erreichbarkeitsanalysen, ergänzt durch die Einbeziehung von demographischen Daten, auch die Durchführung von Interviews einschließt.

Der Beitrag gliedert sich wie folgt: Zunächst werden grundlegende Daten und Informationen zum System der (ambulanten) Pflege sowie zur Situation im Untersuchungsgebiet Unterfranken (Regierungsbezirk) dargelegt (Kapitel 2), bevor anschließend die angewandte Methodik, insbesondere zu raster- und vektorbasierten Erreichbarkeitsanalysen, vorgestellt wird (Kapitel 3). In Kapitel 4 werden die Ergebnisse der Modellierung visuell und argumentativ erläutert sowie bezüglich möglicher Konsequenzen für das Pflegesystem in Unterfranken als wichtigem Bestandteil der regionalen Daseinsvorsorge diskutiert. Abschließend wird die verwendete Methodik kritisch reflektiert, danach ein Fazit gezogen.

\section{Grundlagen, Entwicklungen und die aktuelle Situation der Pflege in Unterfranken}

Die Pflege in Deutschland wird durch die Sozialgesetzbücher (SGB) V und XI geregelt, wobei zwischen Behandlungs- und Grundpflege unterschieden wird. Als "Behandlungspflege“" werden alle an Fachkräfte weitergeleitete Maßnahmen ärztlicher Behandlung verstanden, die dazu dienen, Krankheiten zu heilen, Verschlimmerungen zu verhindern oder Beschwerden zu lindern. Die Unterstützung im Ablauf des täglichen Lebens bei gewöhnlichen und regelmäßig wiederkehrenden Aufgaben wird als "Grundpflege" definiert (Mittelstaedt 1998: 7).

Die Leistungen der Grundpflege werden im SGB XI klar benannt und definiert. Kostenträger der Leistungen sind die Pflegekassen als Träger der Pflegeversicherun- gen nach § 1 SGB XI (Mittelstaedt 1998: 7). Die Behandlungspflege wird nach SGB $V$ abgerechnet, wobei zwischen Krankenhausvermeidungspflege und häuslicher Pflege zur Sicherung der ärztlichen Behandlung zu unterscheiden ist. Des Weiteren müssen die Leistungen ärztlich verordnet sein und es darf keine weitere Person im Haushalt leben, die die Leistungsempfängerin bzw. den Leistungsempfänger in adäquatem Maße versorgen und pflegen kann (§ 37 Abs. 1-3 SGB V).

Der in Kapitel 1 angesprochene Grundsatz „ambulant vor stationär" sowie die Einführung der Pflegeversicherung zum 1. Januar 1995 und der damit verbundenen Erstattung von Leistungen in der häuslichen (ab April 1995) und in der stationären Pflege (ab Juli 1996) resultierte in einem deutlichen Anstieg der ambulanten Pflegedienste von 10.820 (1999) auf 14.050 (2017) für ganz Deutschland, wovon $52 \%$ private Träger haben (Statistisches Bundesamt 2018: 21). Grund für die Zunahme an Pflegediensten war freilich auch ein Anstieg der pflegebedürftigen Menschen. Zwischen 1999 und 2017 erhöhte sich ihre Zahl um 69 \% von 2 auf 3,4 Millionen. Die absolute Zahl der in der häuslichen Umgebung versorgten Pflegebedürftigen stieg dabei überdurchschnittlich stark, um circa $80 \%$, auf insgesamt fast 2,6 Millionen Menschen (StBA 2017). ${ }^{1}$ Es ist jedoch zu berücksichtigen, dass bei Weitem nicht alle zu Hause versorgten Menschen durch ambulante Pflegedienste betreut werden. Angehörige tragen einen Großteil (68 \%) der häuslichen Pflege (Statistisches Bundesamt 2018: 16).

Für den Regierungsbezirk Unterfranken ergibt sich ein Anstieg der ambulanten Pflegedienste von 147 im Jahr 1999 auf 205 im Jahr 2017. Die Anzahl der durch ambulante Pflegedienste versorgten Menschen stieg um $98 \%$, von 6.220 (1999) auf $12.334(2017)^{2}$ und fiel somit noch einmal stärker aus als auf Bundesebene. Die neuesten Zahlen zur Ermittlung der Pflegebedürftigen stammen aus dem Jahr 2017. In diesem Jahr waren 49.061 Personen als pflegebedürftig eingestuft (Stand 1999: 32.793). ${ }^{3}$ Der Anstieg war von 1999 bis 2017 mit

1 Genesis-Online-Datenbank des Statistischen Bundesamtes: Pflegebedürftige nach Art der Versorgung und Altersgruppen, 1999 bis 2017. https://www-genesis.destatis.de/genesis/online (28.05.2020).

2 Genesis-Online-Datenbank des Bayerischen Landesamtes für Statistik: Pflegedienste: Kreis, Pflegedienste, Pflegebedürftige, Personal, Jahre, 1999 bis 2017. https://www.statistikdaten.bayern. de/genesis/online (28.05.2020).

3 Genesis-Online-Datenbank des Bayerischen Landesamtes für Statistik: Pflege: Kreise, Pflegebedürftige nach Art des Pflegeheims, Pflegegeldempfänger, Jahr. https://www.statistikdaten.bayern.de/ genesis/online (28.05.2020). 
$49,6 \%$ folglich gut $20 \%$ geringer als auf der Bundesebene. Für Unterfranken gilt ebenso wie für die Bundesebene die Aussage, dass die Angehörigen den größten Teil der Pflegeleistungen erbringen (49\%). $29 \%$ der Versorgung entfallen auf die vollstationäre, weitere $25 \%$ auf die ambulante Pflege.

Bezogen auf Pflegedienste werden in Unterfranken $54 \%$ der Pflegebedürftigen in vollstationären Einrichtungen ${ }^{4}$ und $46 \%$ durch ambulante Pflegedienste ${ }^{5}$ versorgt. Bei der Interpretation der Werte fällt auf, dass die Anteile zwischen den Arten von Anbietern gleichmäßig verteilt sind. $54 \%$ sind vollstationäre Einrichtungen, die $29 \%$ der Pflegebedürftigen versorgen, während ambulante Pflegedienste $46 \%$ aller Einrichtungen ausmachen und mit $25 \%$ einen etwas geringeren Anteil der Pflegebedürftigen betreuen als vollstationäre. Da keine nach Träger der ambulanten Pflegedienste aufgeschlüsselten Daten für Unterfranken vorliegen, können darüber auch keine verlässlichen Aussagen getroffen werden. Bei einer Orientierung am bundesweiten Durchschnitt hätten knapp über 100 der 205 unterfränkischen ambulanten Pflegedienste private Träger.

\section{Methodik}

In dieser Untersuchung wurde ein Mix aus verschiedenen Methoden angewendet. Die unterschiedlichen methodischen Bestandteile, welche neben der GISModellierung auch die Aufbereitung und Einbeziehung verschiedener Datensätze sowie die implizite Berücksichtigung der Interviewinhalte beinhalten, werden nachfolgend kurz beschrieben.

\subsection{Demographische Informationen und Bevölkerungsvorausberechnung}

Um den Erreichbarkeitsmodellierungen größere Aussagekraft zu verleihen, werden demographische Informationen in die rasterbasierten Erreichbarkeitsanalysen einbezogen. Die Einbeziehung von kleinräumig differenzierten Bevölkerungsdaten ist für die Untersuchung

4 Genesis-Online-Datenbank des Bayerischen Landesamtes für Statistik: Pflegeheime: Kreis, Pflegeheime, verfügbare Plätze, Pflegebedürftige, Personal, Jahr. https://www.statistikdaten. bayern.de/genesis/online (28.05.2020).

5 Genesis-Online-Datenbank des Bayerischen Landesamtes für Statistik: Pflegedienste: Kreis, Pflegedienste, Pflegebedürftige, Personal, Jahre, 1999 bis 2017. https://www.statistikdaten.bayern. de/genesis/online (28.05.2020). erstrebenswert, da die vorrangige Zielgruppe ambulanter Pflegeleistungen - vor allem ältere Menschen nicht gleichmäßig über den Untersuchungsraum verteilt lebt und sich in Zukunft nicht überall mit der gleichen Dynamik entwickeln wird.

Es wurde alleinig die Altersgruppe „80 Jahre und älter" als demographischer Input für alle Berechnungen ausgewählt, um damit vereinfacht das Potenzial pflegebedürftiger Menschen in die Modellierung mit einzubeziehen. Die Wahl dieser Altersgruppe lässt sich dadurch begründen, dass diese mit 52,1\% die mit Abstand größte Gruppe der Pflegebedürftigen in Deutschland darstellt. Außerdem steigt mit der Altersgruppe der 80- bis 84-Jährigen sowie den darauffolgenden Altersstufen der Anteil der Pflegebedürftigen an den gesetzlich Versicherten, also das Pflegerisiko, vergleichsweise stark an (Schwinger/Tsiasioti 2018: 175 f.). Zudem kann durch die Wahl der Altersklasse „80 Jahre und älter" auch die Kompatibilität mit den notwendigen Zensusdaten, welche nur Altersgruppen in 10er-Abstufungen beinhalten, sichergestellt werden.

Als räumliche Grundlage (Referenzraster) zur Verortung der Bevölkerungsinformationen dienen die 100x100m-Rasterzellen aus dem Zensus 2011, der einer Bevölkerungs-, Gebäude- und Wohnungszählung entspricht. ${ }^{6}$

Um die Werte in den Rasterzellen für die Personen „80 Jahre und älter" auch entsprechend für den aktuellen Stand (2017) sowie für die prognostizierte Entwicklung (2025) ,hochzurechnen', müssen entsprechende Faktoren zur Multiplikation gebildet werden. Wie bei der Umrechnung durch den Korrekturfaktor, müssen auch hierfür gleiche Verhältnisse für alle Rasterzellen einer Gemeinde angenommen werden. Die Faktoren zur Veränderung der Anzahl der Personen zwischen 2011 und 2017 sowie bis 2025 werden auf der Basis der Gemeindesummen des Zensus 2011, der Fortschreibung des Bevölkerungsstandes 2017 sowie der Bevölkerungsvorausberechnung des Demographie-Spiegels 20142028/2034 für das Jahr 2025 gebildet. Die beiden letzteren Datensätze wurden vom Bayerischen Landesamt für Statistik auf Anfrage bereitgestellt und entsprechend zusammengefasst, um so die Altersgruppe „80 Jahre und älter" eigens ausweisen zu können. Das Jahr 2025 als Prognosezeitpunkt zu wählen, begründet sich dadurch, dass dieses noch in Zyklen der aktuellen Pflegebedarfsplanung liegt und Fehler bei gegebenenfalls unzutreffenden demographischen Annahmen nur durch mäßige

6 https://www.zensus2011.de/DE/Home/Aktuelles/DemografischeGrunddaten.html?nn=3065474 (28.05.2020). 
Tabelle 1: Nachweis der fünf Interviewpartnerinnen und -partner (eigene Darstellung)

\begin{tabular}{|c|c|c|}
\hline Interview 1 & 30. November 2018 & $\begin{array}{l}\text { Pflegestützpunkt Würzburg/Kommunalunternehmen des Landkreises Würzburg, } \\
\text { Stabsstelle Senioren }\end{array}$ \\
\hline Interview 2 & 5. Dezember 2018 & Caritasverband für die Diözese Würzburg e.V. \\
\hline Interview 3 & 12. Dezember 2018 & Caritasverband für die Stadt und den Landkreis Würzburg \\
\hline Interview 4 & 9. Januar 2019 & Home Instead Seniorenbetreuung \\
\hline Interview 5 & 6. Februar 2019 & Soleo Aktiv GmbH, Sozialstation Würzburg \\
\hline
\end{tabular}

Abweichungen gekennzeichnet wären. Es gilt bei der Interpretation zu beachten, dass die verwendeten Daten des Demographie-Spiegels noch auf der Fortschreibung des Bevölkerungsstandes zum 31. Dezember 2014 sowie auf dessen demographischen Parametern (Sterblichkeit, Binnenwanderung etc.) beruhen. Darauffolgend können für alle Rasterzellen mit Wohnbevölkerung auf der Basis der korrigierten Ausgangswerte für das Jahr 2011 geschätzte Personenzahlen für die Jahre 2017 und 2025 in der genannten Altersgruppe gebildet werden.

\subsection{Experteninterviews}

Um weiterführende Informationen (vor allem zu Betriebs- und Kontextwissen) zu gewinnen, die bedeutend für die Modellierung der Erreichbarkeiten sowie für die inhaltliche Interpretation der Ergebnisse sind, wurden leitfadengestützte Experteninterviews durchgeführt. Dazu wurden aus den Leitfragen dieser Arbeit Fragenkomplexe mit den Schwerpunkten „Relation Mitarbeiter - Pflegebedürftige“, „Konkrete Tourenplanung“, „Einzugsgebiet nach §11 der Rahmenvereinbarung der ambulanten Pflege“, „Abrechnungspauschalen Fahrzeiten/Distanzen" und "Notrufsituation/Hausnotrufknopf" gebildet, aus denen wiederum insgesamt zwölf Interviewfragen abgeleitet wurden. Die Interviewpartnerinnen und -partner stammen sowohl von freigemeinnützigen als auch von privaten Trägern ambulanter Pflegedienste (vgl. Tabelle 1). Für das Interview mit einem Vertreter einer Gebietskörperschaft wurde ein abweichender Leitfaden verwendet, da es hierbei weniger um die Gewinnung von Betriebswissen als vielmehr um Kontextwissen ging. Dementsprechend wurden hier vorwiegend indirekte Fragen gestellt. Zur Auswertung der fünf Interviews wurden die im Gespräch aufgezeichneten Audioaufnahmen transkribiert.

\subsection{Standorte ambulanter Pflegedienste}

In die Analyse wurden Standorte ambulanter Pflegedienste im Regierungsbezirk Unterfranken sowie - um Grenzeffekte bei der Erreichbarkeitsanalyse zu vermeiden - außerhalb Unterfrankens im Umkreis von 15 Kilometern zu unterfränkischen Grenzgemeinden, welche Leistungen nach SGB V und SGB XI erbringen, aufgenommen. Diese wurden durch postleitzahlgenaue Suchläufe in den Suchmaschinen der AOK (Pflege-Navigator) ${ }^{7}$ und des Verbandes der Ersatzkassen e.V. (Pflegelotse) ${ }^{8}$ ermittelt. Zusätzlich wurden als Metadaten die Anzahl der aktuell versorgten Patientinnen und Patienten aus den Transparenzberichten 2017 und 2018 der Pflegedienste ergänzt. Stark spezialisierte Pflegedienste, wie etwa Heimbeatmungsservice oder Kinderpflege, wurden aufgrund der Fragestellung nicht beachtet. Die Standorte wurden mittels QGIS über OpenStreetMap-Adressdaten geocodiert und bei Abweichung nachträglich korrigiert. Insgesamt konnten 189 Standorte ambulanter Pflege innerhalb des Untersuchungsgebietes und 90 in den angrenzenden Bundesländern und bayerischen Landkreisen geocodiert werden.

\subsubsection{Erreichbarkeitsanalysen}

Für diese Studie wird sowohl eine vektor- als auch eine rasterbasierte Erreichbarkeitsanalyse durchgeführt. Beide "weisen fachspezifische Vor- und Nachteile" auf (Rauch/Rauh 2016: 443). Für die vektorbasierte Erreichbarkeitsanalyse ist aufgrund des geringen Datenaufwands eine schnelle Berechnung klarer Einzugsgebiete möglich. Das Modell ist jedoch aufgrund der statischen Isochronen nur wenig flexibel. Die rasterbasierte Erreichbarkeitsanalyse ermöglicht eine stufenlose, dis-

$7 \mathrm{https}: / / \mathrm{www}$.pflege-navigator.de/index.php?module=careservice (28.05.2020).

8 https://www.pflegelotse.de (28.05.2020). 
Tabelle 2: Geschwindigkeiten für verschiedene Straßentypen, die über das Erreichbarkeitsmodell des BBSR hinaus mit den Daten aus dem Netzmodell des DLM 250 in inner- und außerorts differenziert wurden

\begin{tabular}{lll}
\hline Straßentyp/Funktion & Geschwindigkeit innerorts (in $\mathbf{~ m} / \mathbf{h})$ & Geschwindigkeit außerorts (in $\mathbf{~ m} / \mathbf{h}$ ) \\
\hline Autobahn & 70 & 100 \\
Bundesstraße & 40 & 75 \\
Landes-, Staats- oder Kreisstraße & 40 & 70 \\
Ortsverbindungsstraße bzw. Durchfahrtsstraße & 40 & 60 \\
Fernstraße/Bundesstraße, autobahnähnliche & 40 & 85 \\
Straße & & \\
Gemeindestraße & 30 & 35 \\
\hline
\end{tabular}

krete Raumdarstellung und Verknüpfungen mit weiteren Metadaten sowie gute Differenzierungsmöglichkeiten. Diese Methode erfordert jedoch einen deutlich höheren Rechenaufwand und liefert keine klaren Einzugsgebiete.

In dieser Studie sollen anhand der vektorbasierten Erreichbarkeitsanalyse konkrete Einzugsbereiche der Pflegestandorte, begrenzt durch die vergüteten Anfahrtspauschalen nach SGB V und SGB XI und die durch die Fahrtzeiten entstehenden Personalkosten, ermittelt werden. Anhand der rasterbasierten Erreichbarkeitsanalyse soll ein Gesamtüberblick über die Erreichbarkeitsverhältnisse von Standorten ambulanter Pflegedienste im Untersuchungsgebiet entstehen. Die Verknüpfung demographischer Daten ermöglicht bei dieser Methode zusätzlich eine differenzierte Analyse anhand der Altersstruktur und ermöglicht es, Aussagen über die potenzielle Veränderung der Anzahl der Pflegekundinnen und -kunden in Unterfranken zu treffen.

\subsubsection{Aufbereitung des Straßennetzwerks}

Die Grundlage für die Erreichbarkeitsanalyse bilden Straßendaten von OpenStreetMap, da diese eine hohe Auflösung bieten und flächendeckend zur Verfügung stehen. Das zugrunde liegende Straßennetz wurde auf das Gebiet Unterfrankens mit einem Puffer von 15 Kilometer um die Gebietsgrenze beschränkt. Da für die Beantwortung der Fragestellung ein großer Maßstab gewählt wurde und die demographischen Daten auf ein $100 \times 100 \mathrm{~m}$-Raster übertragen wurden, hat sich für die Straßendaten eine Unterteilung in inner- und außerorts empfohlen. Dies ermöglicht eine kleinteiligere Zuordnung der Geschwindigkeiten und verbessert somit das Routing. Die Differenzierung der Straßendaten in innerund außerorts fand unter Zuhilfenahme des Digitalen Landschaftsmodells 1:250.000 (DLM 250) statt.
Bei den Straßendaten von OpenStreetMap handelt es sich um Vektordaten. Diese sind in Straßenabschnitte unterteilt, die wiederum nach der Funktion der Straße klassifiziert sind. Für jede Klasse, 25 insgesamt, wurde eine spezifische Geschwindigkeit festgelegt (Dahlgren 2008: 17). Die Geschwindigkeiten ergaben sich vorrangig aus dem Erreichbarkeitsmodell des Bundesinstituts für Bau-, Stadt- und Raumforschung (BBSR) sowie ergänzend aus dem Netzmodell des DLM 250 (vgl. Schwarze/ Spiekermann 2019). Die nach dem DLM 250 angepassten Geschwindigkeiten sind in Tabelle 2 aufgelistet.

Abschließend wurde mithilfe von ESRI ArcGIS [10] aus den Daten von OpenStreetMap ein routingfähiges Netzwerk erstellt, bestehend aus Knoten und Kanten, mit dem die weiterführenden Erreichbarkeitsanalysen durchgeführt werden konnten.

\subsubsection{Rasterbasierte Erreichbarkeitsanalyse}

Der rasterbasierten Erreichbarkeitsanalyse wurde das ETRS89-LAEA 100m-Raster des Bundesamtes für Kartographie und Geodäsie zugrunde gelegt. ${ }^{9}$ Den Rasterzellen wurde als Attributinformation jeweils ein Bevölkerungswert aus den gitterzellenbasierten Ergebnissen des Zensus 2011 zugeordnet. Die gitterbasierten Daten wurden wie in Kapitel 3.1 beschrieben aufbereitet. Die Fortschreibung und Vorausberechnung der rasterbasierten Bevölkerungsdaten wird ebenfalls in Kapitel 3.1 genauer erläutert.

Auf der Grundlage der Rasterzellenmittelpunkte, des aufbereiteten Verkehrswegenetzes und der geocodierten Standorte ambulanter Pflegedienste wurde für jeden Rasterzellenmittelpunkt im Untersuchungsgebiet

9 https://www.bkg.bund.de/SharedDocs/Produktinformationen/ BKG/DE/P-2020/200310_GeoGitter.html (28.05.2020). 
die Fahrzeit vom zeitlich nächstgelegenen Pflegestandort ermittelt. Dabei wird die Annahme getroffen, dass einem Pflegestandort jeweils die zeitlich nächstgelegenen Rasterzellen bei Wahl der jeweils schnellsten Wegstrecke zugeordnet werden, ein ambulanter Pflegedienst also jene Standorte bedient, zu denen die Fahrzeit am geringsten ist (Nearest-Neighbour-Annahme) (Neumeier 2015: 22).

Die Berechnung der Fahrzeiten erfolgte auf der Basis der Start-Ziel-Kostenmatrix-Analyse mittels der ArcGIS Network-Analyst-Erweiterung. „Die Start-Ziel-Kostenmatrix sucht und misst die kostengünstigsten Routen im Netzwerk von mehreren Startpunkten zu mehreren Zielen. ${ }^{.10}$ Für die Berechnung wurden als Startpunkte die geocodierten Pflegestandorte gewählt. Als Kostenfaktor wurde die Fahrzeit auf der Basis des erstellten Verkehrswegenetzes gewählt. Somit wurden von jedem Standort eines ambulanten Pflegedienstes aus jeweils die Fahrzeiten zu jedem Zielpunkt im Untersuchungsgebiet, in diesem Fall konkret zu den mit Bevölkerungsinformationen versehenen Rastermittelpunkten des zugrunde liegenden 100-Meter-Rasters, berechnet. Mithilfe der Summarize-Funktion wurde nachgehend für jede Rasterzelle die geringste Fahrzeit ermittelt.

Die Start-Ziel-Kostenmatrix-Analyse wurde für alle im Untersuchungsgebiet liegenden 55.955 Rasterzellen, die Bevölkerungsinformationen enthalten, durchgeführt. Dabei konnte für $46(0,08 \%)$ Rasterzellen keine Fahrzeit ermittelt werden. Mittels einer Nearest-NeighbourInterpolation wurde den Rasterzellen, bei denen die Fahrzeitenberechnung fehlschlug, die Fahrzeit einer benachbarten Zelle zugeordnet. Fehlerhafte Berechnungen einzelner Rasterzellen sind dabei auf topologische Fehler im Straßennetz zurückzuführen.

\subsubsection{Vektorbasierte Erreichbarkeitsanalyse}

Zweck der vektorbasierten Erreichbarkeitsanalyse ist die Untersuchung der Anfahrtspauschale als Instrument der Fahrtenvergütung. Hierfür wurden die Standorte der ambulanten Pflegedienste sowie das routingfähige Netzwerk benötigt und per Network Analyst in ArcGIS verarbeitet.

Für die vorliegende Untersuchung wurden Zeitgrenzen genutzt, die die Reichweite von Fahrten durch Pflegepersonal, unter Berücksichtigung der vergüteten Anfahrtspauschalen und während der Fahrzeit entste-

$10 \mathrm{https} / / /$ desktop.arcgis.com/de/arcmap/latest/extensions/network-analyst/od-cost-matrix.htm (28.05.2020). henden Personalkosten, angeben. Die hierzu benötigten Parameter sind Anfahrtspauschale und Gehälter des Pflegepersonals. Die Anfahrtspauschale wird in Vergütungsvereinbarungen festgelegt, die zwischen den Trägern der ambulanten Pflegedienste und den Pflegekassen geschlossen werden. Diese sind beispielsweise bei der AOK Bayern einsehbar. Weiterhin gibt es gesonderte Vergütungsvereinbarungen für Leistungen nach SGB V oder SGB XI. Auch die Anfahrtspauschale eines Trägers oder Trägerverbandes ist nicht einheitlich, sondern wird nach Zeitfenstern unterteilt, vorwiegend in 8-20 Uhr und 20-8 Uhr. Da davon auszugehen ist, dass der Großteil der Pflegeleistungen innerhalb des ersten Zeitfensters erbracht wird, wurde dieses für die Modellierung gewählt.

Bei dem Pflegepersonal wurden drei Qualifikationsstufen unterschieden: „Pflegefachkräfte“, „Pflegefachhelfer/-innen“ und „Hauswirtschaftskräfte“. Mithilfe der Unterscheidung nach Qualifikation können bessere Aussagen getroffen werden, welche Spektren der Leistungserbringung in einem bestimmten Bereich abgedeckt sind. Da es keine einheitliche Regelung zur Vergütung von Pflegepersonal gibt, wurde als Grundlage eine Empfehlung des Berufsverbandes für Pflegeberufe Südost genutzt, die sich an dem Tarifvertrag für den öffentlichen Dienst - Pflege (TVöD-P) orientiert. ${ }^{11}$ Da eine Betrachtung der Kosten für eine Anfahrt eines Dienstes nur aus der Sicht des Arbeitgebers Sinn hat, wurden auf den im Tarifvertrag festgesetzten Bruttolohn die Abgaben für Sozialversicherungen nach Angaben der IHK für München und Oberbayern (2019) ${ }^{12}$ aufgeschlagen. Anschließend wurde mit dem „MindestlohnRechner" des Bundesministeriums für Arbeit und Soziales der Stundenlohn ermittelt. ${ }^{13}$ Somit konnten die Personalkosten als Hauptfaktor für die Kostenbildung einer Fahrt modelliert werden. Weitere implizite Kosten wie Sprit, Verschleiß des Fahrzeugs oder Anschaffungskosten wurden im Rahmen der Untersuchung nicht berücksichtigt. Die Formel zur Berechnung der Fahrzeitgrenzen lautet wie folgt:

$$
\left(\frac{\text { Anfahrtspauschale }}{\text { Stundenlohn }(\text { Arbeitgeberbrutto })} \times 60\right) \div 2
$$

11 Auskunft des Berufsverbandes für Pflegeberufe Südost über die Empfehlung zur Vergütung von Pflegepersonal anhand des Tarifvertrags für den öffentlichen Dienst - Pflege vom 8. Januar 2019.

12 https://www.ihk-muenchen.de/de/Service/Recht-und-Steuern/ Arbeitsrecht/\#st_text_picture_19 (28.05.2020).

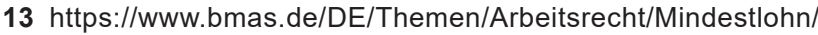
Rechner/mindestlohnrechner.html (28.05.2020). 
Tabelle 3: Isochronengrenzen nach Qualifikationen

\begin{tabular}{lll}
\hline Qualifikation & Fahrzeit in Minuten & \\
\hline & nach SGB V & nach SGB XI \\
\hline Pflegefachkraft & 7,35 & 6,4 \\
Pflegefachhelfer/-in & 8,77 & 7,63 \\
Hauswirtschaftskraft & nicht abgedeckt & 8,94 \\
\hline
\end{tabular}

Anmerkung: Hauswirtschaftskräfte können nur nach SGB XI abgerechnet werden, daher ist die Zelle für SGB V mit „nicht abgedeckt" versehen.

Die Division der Anfahrtspauschale durch den Stundenlohn (Arbeitgeberbrutto) ermittelt den Anteil der Pauschale am Stundenlohn. Dieser Anteil wird durch die Multiplikation mit 60 nun auf einen Minutensatz übertragen. Der abschließende Wert wird nun durch 2 geteilt, um auch die Rückfahrt mit der Anfahrtspauschale abzudecken. Aus der Berechnung ergeben sich Isochronengrenzen (vgl. Tabelle 3).

\section{Ergebnisse}

Im Folgenden werden die Ergebnisse aus der vektor- und rasterbasierten Analyse zur Erreichbarkeit von ambulanten Pflegediensten nach SGB V und SGB XI sowie die räumliche und demographische Differenzierung dieser dargestellt. Die Visualisierung erfolgt insbesondere über Karten der Erreichbarkeitsverhältnisse und graphische Darstellungen statistischer Parameter.

\subsection{Rasterbasierte Erreichbarkeitsanalyse}

Die durchgeführte Start-Ziel-Kostenmatrix-Analyse bezieht sich auf insgesamt 55.955 Rasterzellen mit einem Bevölkerungswert von mindestens einer Person im Untersuchungsgebiet. Hier konnten im Rahmen der Analyse insgesamt für 55.909 Rasterzellen die Fahrzeiten zum jeweils nächstgelegenen Pflegestandort ermittelt werden. Für die Rasterzellen, bei denen die Fahrzeitberechnung fehlschlug, wurden die Fahrzeit mittels einer Nearest-Neighbour-Interpolation berechnet.

Auffällig ist, dass die Fahrzeiten zum nächstgelegenen ambulanten Pflegedienst in den kreisfreien Städten Würzburg, Schweinfurt und Aschaffenburg und in deren direkter Umgebung eher gering sind und sich im Bereich von bis zu fünf Minuten bewegen. In den Agglome- rationsräumen in und um die kreisfreien Städte ist die Dichte der ambulanten Pflegedienste deutlich höher als im weiteren Umland. In den Kreisstädten, Kleinstädten oder bevölkerungsstärkeren Gemeinden Unterfrankens sind meist ebenfalls ambulante Pflegedienste ansässig und somit gering Fahrtzeiten von bis zu fünf Minuten feststellbar. In den ländlichen Gemeinden sind Standorte ambulanter Pflegedienste nur vereinzelt vorhanden, dort in der Regel entlang der Hauptverkehrsachsen wie Bundesstraßen oder Autobahnen. Die Fahrtzeiten in ländlichen Gebieten und Gemeinden sind somit aufgrund der geringeren Dichte der ambulanten Pflegedienste stark von den jeweiligen Verkehrsachsen bzw. den festgelegten Geschwindigkeiten aus dem erstellten Straßennetz abhängig. So ergeben sich entlang von Hauptverkehrsachsen geringere Fahrtzeiten, während die Fahrtzeiten in den Zwischenräumen der Verkehrsachsen deutlich ansteigen. Gebiete mit erhöhter Fahrtzeit sind im Landkreis Würzburg vor allem an der westlichen Kreisgrenze (bis zu 15 Minuten) und im Süden (bis zu 13 Minuten) zu verorten. Im Landkreis Kitzingen sind erhöhte Fahrtzeiten vor allem an der östlichen Kreisgrenze um Geiselwind und östlich von Prichsenstadt zu erkennen. Erhöhte Fahrtzeiten wurden ebenfalls für Rasterzellen im Landkreis Schweinfurt östlich von Schweinfurt, und an der nord- und südwestlichen Landkreisgrenze (bis zu 16 Minuten) sowie für Rasterzellen entlang der Landkreisgrenze im Landkreis Haßberge modelliert. Rasterzellen mit besonders hohen Fahrtzeiten sind vorwiegend in den Landkreisen Rhön-Grabfeld, Bad Kissingen, Main-Spessart, Aschaffenburg und Miltenberg zu verorten. Diese liegen vorwiegend im Bereich des Spessarts und der Rhön. Es handelt sich hierbei jedoch nur um wenige einzelne Rasterzellen mit Fahrtzeiten von über 30 Minuten. Exemplarisch für die Situation in Unterfranken sind in den Abbildungen 1 und 2 die Erreichbarkeitsverhältnisse für die Stadt Würzburg, die Landkreise Würzburg und Main-Spessart abgebildet.

Für die ermittelten Fahrtzeiten ergibt sich ein Median bei rund 4,8 Minuten. Die mittleren $50 \%$ der Daten liegen im Bereich zwischen 2,5 (1. Quartil) und 7,5 (3. Quartil) Minuten. Innerhalb der Antennen (0 bis 14,8 Minuten) liegen rund 98,7\% der ermittelten Fahrtzeiten. Fahrtzeiten, die den Wert von 14,8 Minuten übersteigen, können als potenzielle Ausreißer betrachtet werden. Dies betrifft 731 Fälle, wovon 22 als extreme Ausreißer (Stern), ab einer Fahrtzeit von 22,2 Minuten, angesehen werden können (Eckey/Kosfeld/Türck 2008: 96 ff.; vgl. Abbildung 3).

Auf der Basis dieser Erreichbarkeitsanalyse ergibt sich für die berechneten Fahrtzeiten ein Mittelwert von 


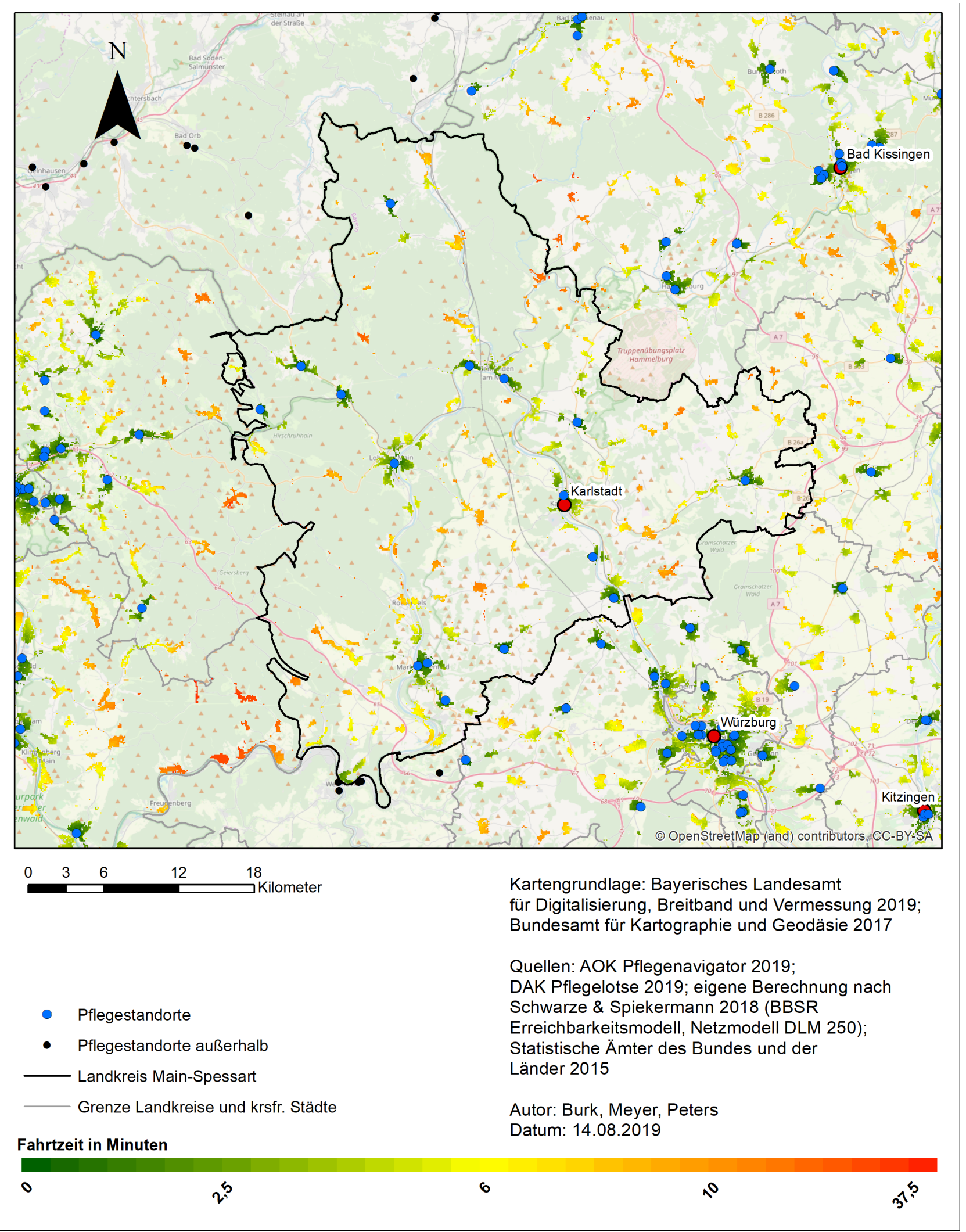

Abbildung 1: Modellierte Erreichbarkeiten von Standorten ambulanter Pflegedienste auf der Basis eines 100m-Rasters im Landkreis Main-Spessart 


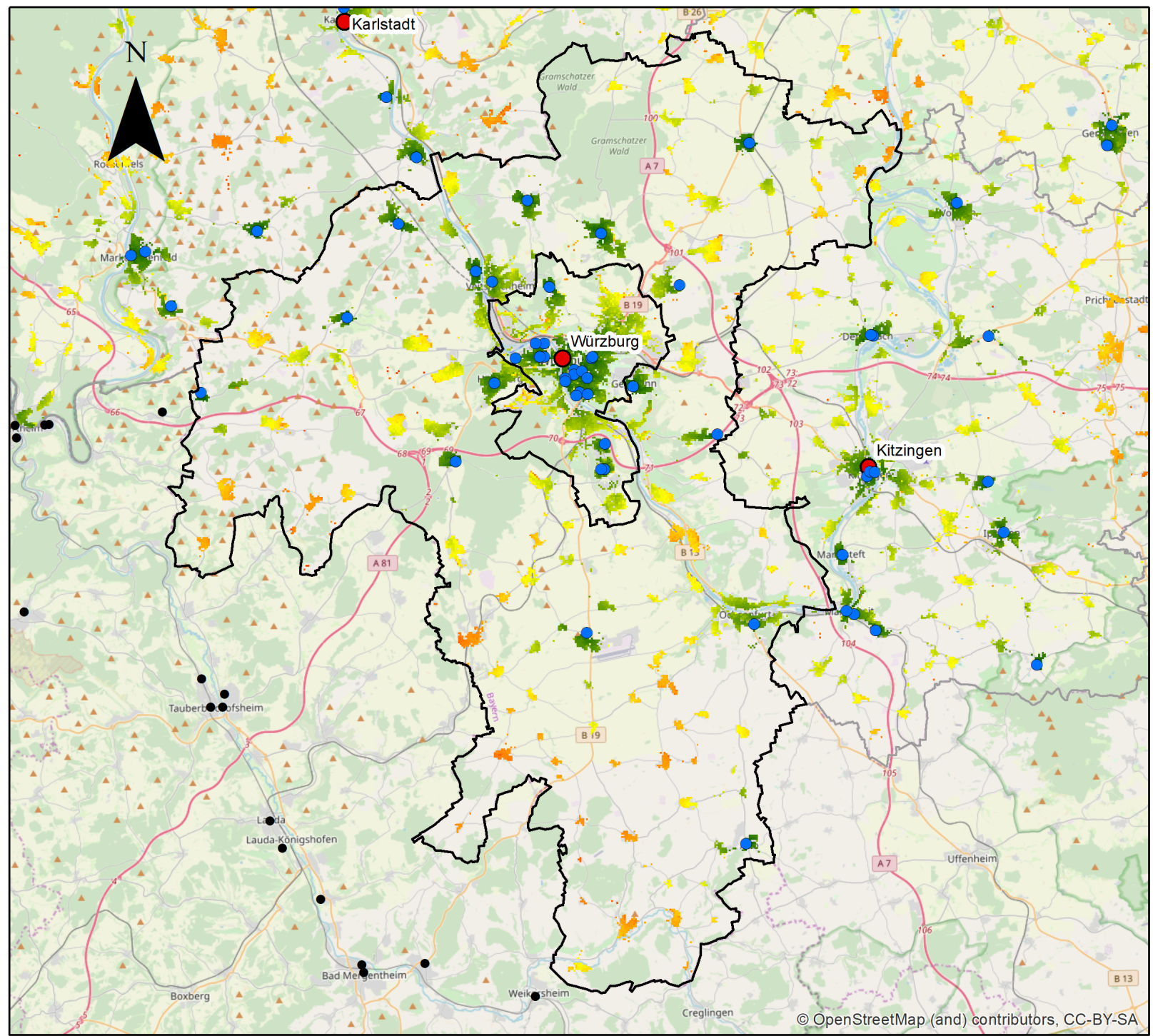

$\begin{array}{lll}0 & 2,5 & 5\end{array}$

10

15

Kilometer

- Pflegestandorte

- Pflegestandorte außerhalb

Würzburg Stadt und Landkreis

Grenze Landkreise und krsfr. Städte

\section{Fahrtzeit in Minuten}

$\eta^{5}$
Kartengrundlage: Bayerisches Landesamt

für Digitalisierung, Breitband und Vermessung 2019;

Bundesamt für Kartographie und Geodäsie 2017

Quellen: AOK Pflegenavigator 2019;

DAK Pflegelotse 2019; eigene Berechnung nach

Schwarze \& Spiekermann 2018 (BBSR

Erreichbarkeitsmodell, Netzmodell DLM 250);

Statistische Ämter des Bundes und der

Länder 2015

Autor: Burk, Meyer, Peters

Datum: 14.08.2019

0

6

$\wedge^{\circ}$

Abbildung 2: Modellierte Erreichbarkeiten von Standorten ambulanter Pflegedienste auf der Basis eines 100m-Rasters in Stadt und Landkreis Würzburg 


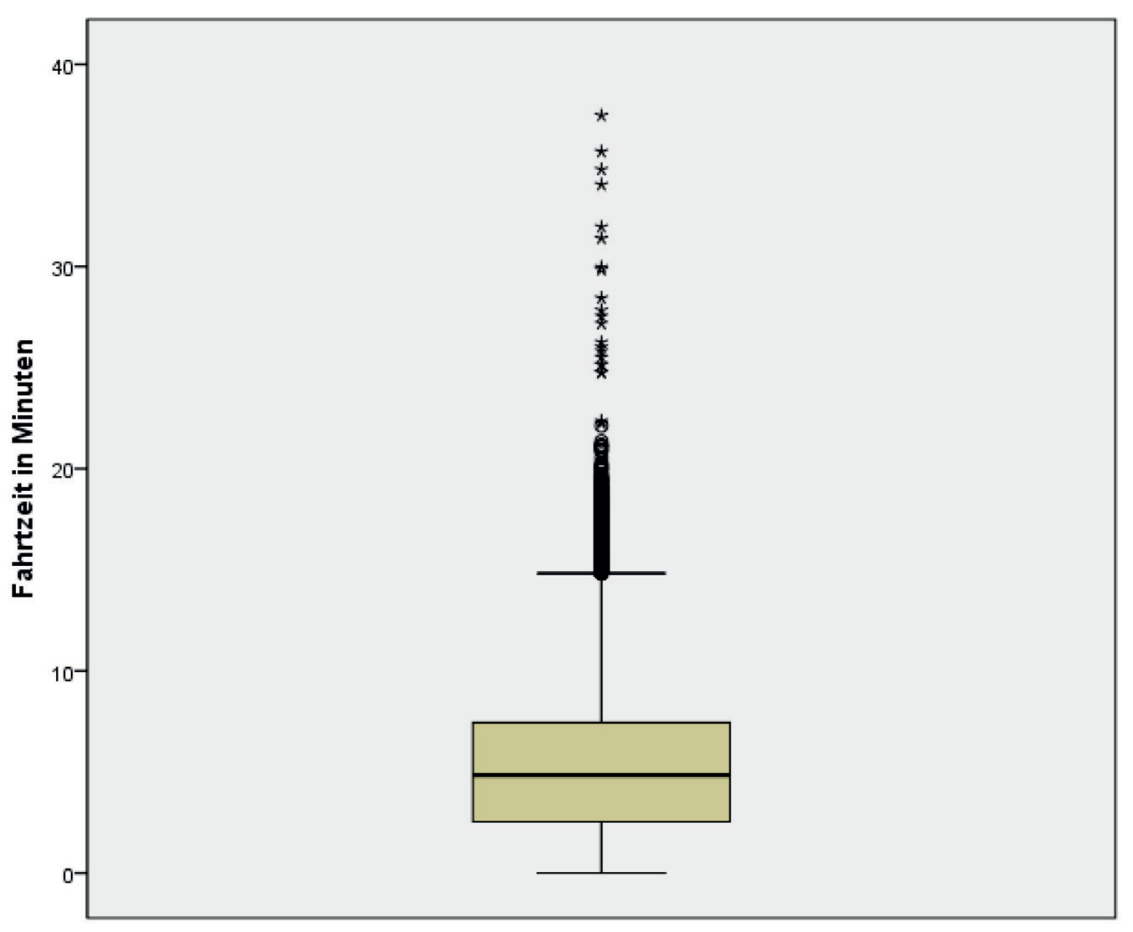

Abbildung 3: Boxplot: Modellierte Erreichbarkeiten von Standorten ambulanter Pflegedienste auf der Basis des 100m-Rasters in Unterfranken

rund 5,3 Minuten bei einer Standardabweichung von rund 3,5 Minuten. Die maximale Fahrtzeit beträgt 37,5 Minuten. Die Rasterzelle mit dem entsprechend hohen Wert liegt im Schondratal im Landkreis Bad Kissingen. In Abbildung 4 ist ein starker Anstieg der Häufigkeiten im Fahrtzeitenbereich von 0 bis etwa 3 Minuten zu erkennen. Mit höherer Fahrtzeit nehmen die Häufigkeiten bis zu etwa 15 Minuten Fahrtzeit relativ stark ab. Zwischen 15 und 17 Minuten ist ein leichter Anstieg der Häufigkeiten zu beobachten. Ab etwa 18 Minuten Fahrtzeit ist eine erneute Abnahme zu erkennen. Die Häufigkeiten bewegen sich ab 20 Minuten Fahrtzeit auf einem sehr geringen Niveau.

\subsection{Vektorbasierte Erreichbarkeitsanalyse}

In Abbildung 5 sind Fahrzeitzonen dargestellt, die die maximalen Reichweiten für Pflegepersonal unterschiedlicher Qualifikationsstufen angeben, die durch die Anfahrtspauschale nach SGB $\vee$ abgedeckt sind. Es wird zwischen Pflegefachkräften (mit einer maximalen Reichweite von 7,4 Minuten) und Pflegefachhelferinnen/-helfer (Reichweite von 8,8 Minuten) unterschieden. Des Weiteren sind alle Standorte ambulanter Pflegedienste in
Unterfranken dargestellt, die die Ausgangspunkte für die Fahrzeitzonen bilden. Ebenso sind die Standorte in den an Unterfranken angrenzenden Kreisen dargestellt, um Grenzeffekte in die Untersuchung miteinzubeziehen. Zur besseren Übersicht wurden die Fahrzeitzonen der Standorte außerhalb Unterfrankens auf ihre Auswirkungen in Unterfranken beschränkt.

In den Städten und angrenzenden Landkreisen Würzburg, Aschaffenburg, Schweinfurt und Kitzingen ist die Erreichbarkeit flächendeckend garantiert. Dies gilt ebenso für die Städte Haßfurt, Bad Kissingen, Bad Neustadt, Karlstadt und Miltenberg. Deutlich zu erkennen ist eine schlechte Erreichbarkeit im westlichen Landkreis Main-Spessart, dem östlichen Landkreis Aschaffenburg sowie im Nordwesten der Landkreise Bad Kissingen und Rhön-Grabfeld. Dies ist durch die Topographie zu erklären, da die Lücken deckungsgleich mit den Mittelgebirgen Spessart und Südrhön sind. Die gute Erreichbarkeit in den Städten und ihrer unmittelbaren Umgebung resultieren aus der höheren Einwohnerzahl bzw. Einwohnerdichte und dem damit verbundenen erhöhten „Pflegebedürftigenpotenzial“. Die hohe Anzahl an Diensten in diesen Bereichen deckt sich auch mit den Angaben der Interviewpartner (Interview 12018: 3; Interview 2 2018: 5).

In der Abbildung 5 sind darüber hinaus Verkehrsachsen zu erkennen, entlang derer die Fahrzeitzonen 


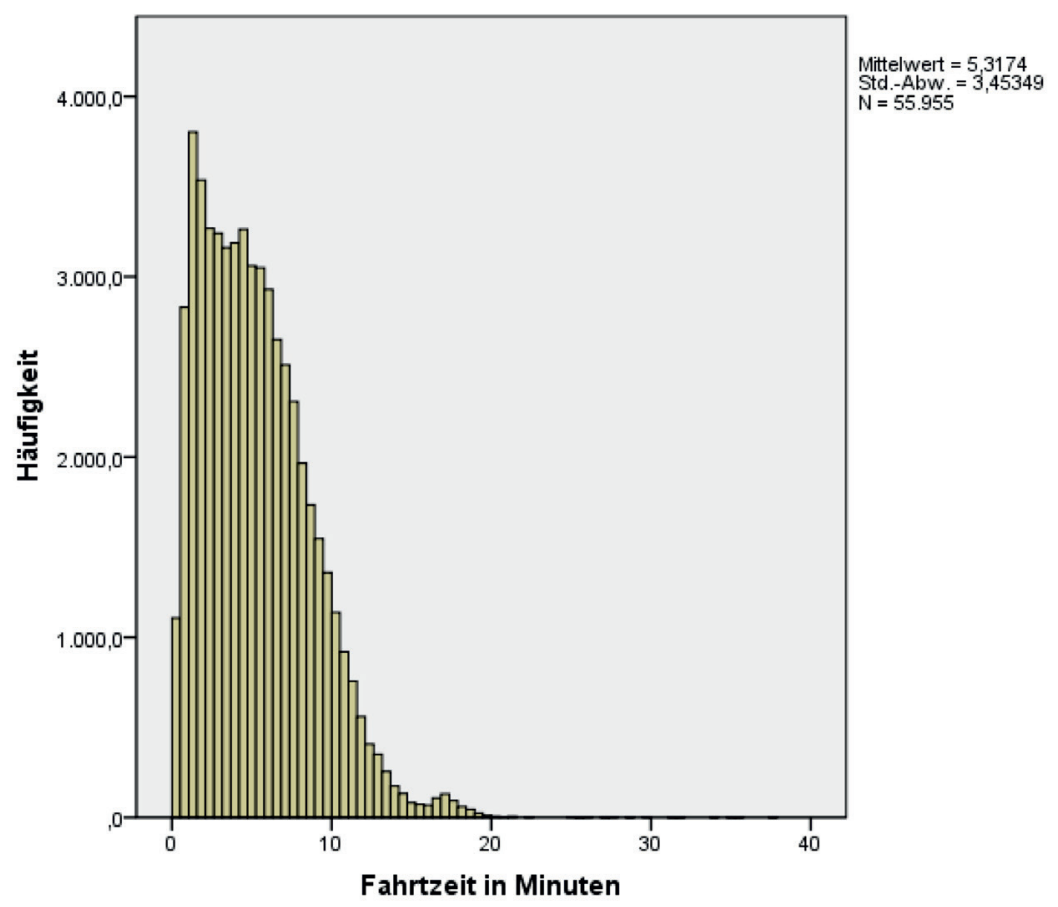

Abbildung 4: Histogramm: Modellierte Erreichbarkeiten von Standorten ambulanter Pflegedienste auf der Basis des 100m-Rasters in Unterfranken

verlaufen. Am deutlichsten sind dies die A 7 zwischen Würzburg und Schweinfurt, die A 3 zwischen Kitzingen und Marktheidenfeld sowie der Main mit Land- bzw. Bundesstraßen auf beiden Seiten zwischen Aschaffenburg und Miltenberg sowie zwischen Karlstadt und Kitzingen. Letzteres ist auch auf eine Konzentration von Ortschaften entlang des Mains zurückzuführen.

In Abbildung 6 werden ähnliche Inhalte dargestellt wie in Abbildung 5, mit dem Unterschied, dass die Fahrzeitzonen hier mit den Anfahrtspauschalen nach SGB XI berechnet wurden. Darüber hinaus wurde eine weitere Fahrzeitzone ergänzt, da seit 2015 nach SGB XI auch Leistungen der Hauswirtschaft abrechenbar sind (Interview 3 2018: 8; Interview 2 2018: 3). Aufgrund der niedrigeren Anfahrtspauschalen der Versorgungsverträge für das SGB XI fallen auch die Fahrzeitzonen kleiner aus: Pflegefachkraft (6,4 Minuten), Pflegefachhelferin/-helfer (7,6 Minuten), Hauswirtschaftskraft (8,9 Minuten).

Somit verkleinert sich auch die Zone, in der eine Versorgung durch Fachkräfte rentabel ist. Gebiete, die nach SGB $\vee$ noch von Pflegefachhelferinnen/-helfern abgedeckt werden konnten, sind somit nach SGB XI nur noch für die Anfahrt von Hauswirtschaftskräften rentabel. Diese Bereiche sind besonders deutlich entlang der A 7 zwischen Würzburg und Schweinfurt sowie im südöstlichen Landkreis Schweinfurt und im Landkreis Kitzin- gen zu erkennen. Die Erreichbarkeiten der ambulanten Pflegedienste, ausgehend von den Standorten, sind also besonders gut in und rund um die größeren Städte in Unterfranken sowie entlang bestimmter Verkehrsachsen. Besonders interessant bei der Betrachtung sind die Fahrzeitzonen der Pflegefachhelferinnen/-helfer. Hierbei handelt es sich um angelernte Kräfte, die einen Großteil der Leistungen erbringen und somit in der Praxis den Großteil des Personals eines Pflegedienstes bilden (Interview 3 2018: 1). Die Pflegefachkräfte werden hingegen häufig in der Pflegedienstleistung und -planung eingesetzt (Interview 4 2019: 2).

\subsection{Räumliche und demographische Differenzierung der Erreichbarkeitsverhältnisse}

Die zuvor vorgestellten Erreichbarkeitsverhältnisse durch ambulante Pflegedienste lassen sich im Folgenden noch genauer räumlich (nach Landkreisen) und demographisch sowie als statistische Angaben auf der Basis der Zuordnung zu den Pflegediensten differenzieren. Hierzu ist es allerdings notwendig, die Bevölkerungsentwicklung in den Gemeinden Unterfrankens nachzuvollziehen, da dadurch die Entwicklung des Pfle- 


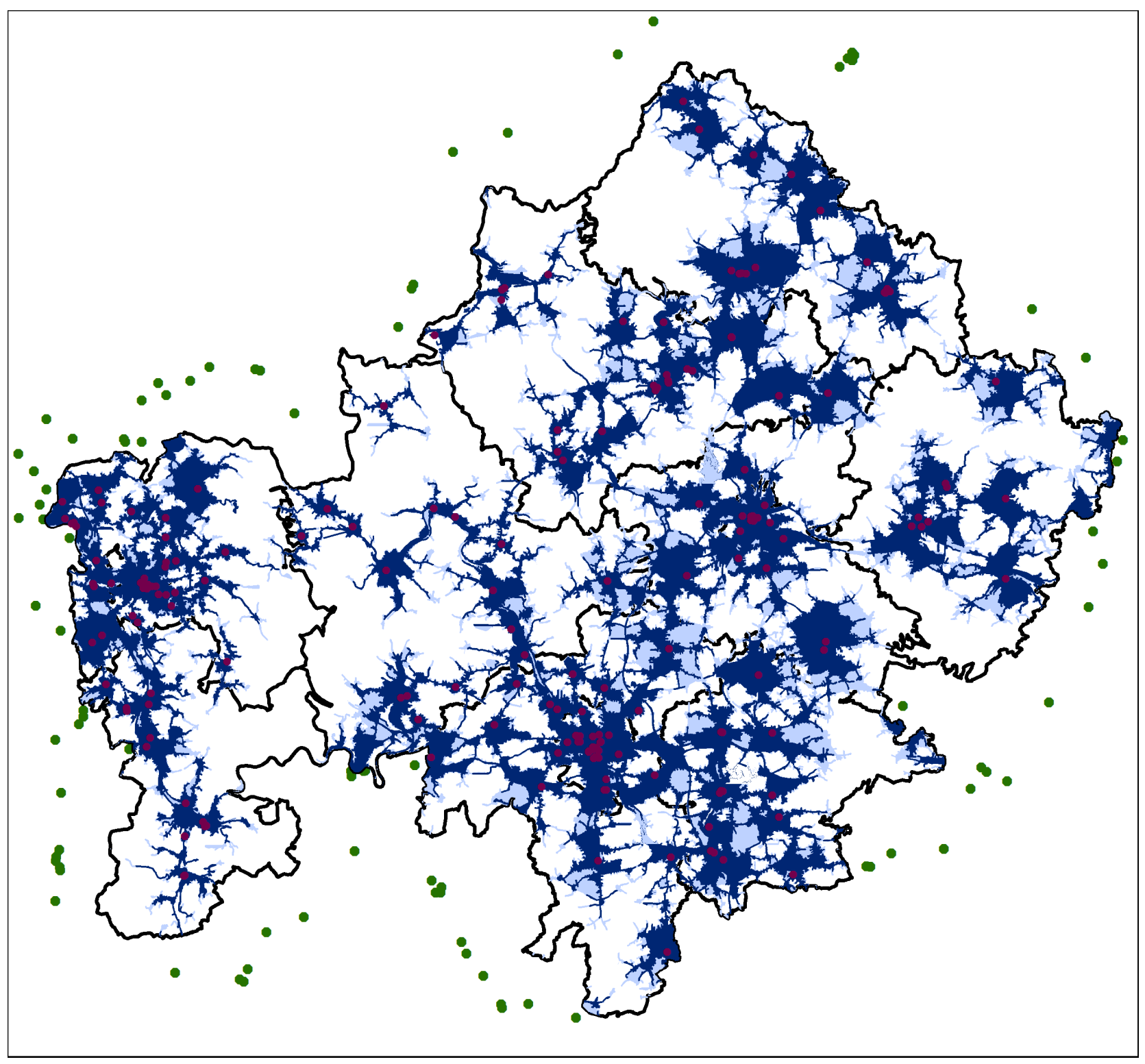

\section{$\begin{array}{llllll}0 & 5 & 10 & 20 & 30 & 40\end{array}$}

Kilometer

- Pflegestandorte

- Pflegestandorte außerhalb Unterfrankens

Grenze Landkreise und krsfr. Städte

Fahrzeitzonen

7,4 Minuten (Pflegefachkraft)

8,8 Minuten (Pflegefachhelferin)
Kartengrundlage: Bayerisches Landesamt für Digitalisierung, Breitband und Vermessung 2019; Bundesamt für Kartographie und Geodäsie 2017

Quellen: AOK Pflegenavigator 2019;

DAK Pflegelotse 2019; eigene Berechnungen nach Schwarze \& Spiekermann 2018 (BBSR

Erreichbarkeitsmodell, Netzmodell DLM 250); sowie eigene Berechnungen nach Tarifvertrag für den öffentlichen Dienst - Pflege (vom 13. September 2005, letzte Änderung 18. April 2018) und Versorgungsverträge der Träger ambulanter Pflege mit den Pflegekassen (AOK Bayern 2019)

Autoren: Burk, Meyer, Peters

Datum: 06.02.2019 


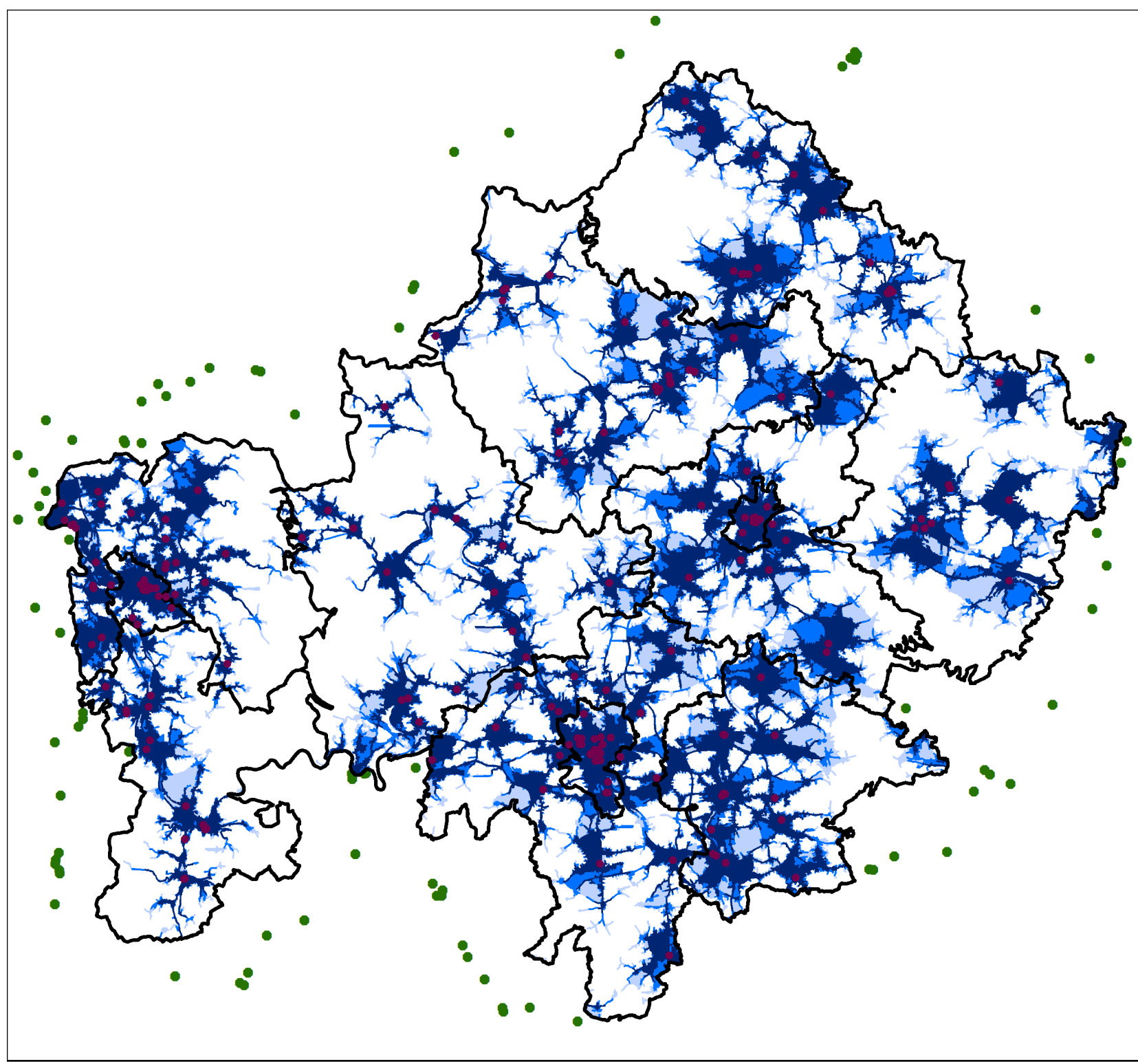

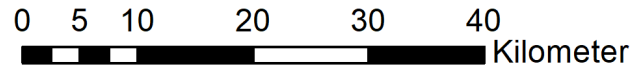

- Pflegestandorte

- Pflegestandorte außerhalb Unterfrankens Grenze Landkreise und krsfr. Städte

\section{Fahrzeitzonen}

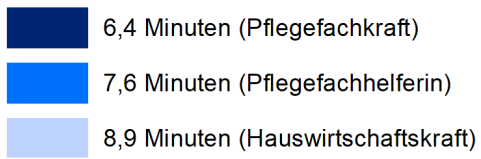

Kartengrundlage: Bayerisches Landesamt für Digitalisierung, Breitband und Vermessung 2019; Bundesamt für Kartographie und Geodäsie 2017

Quellen: AOK Pflegenavigator 2019;

DAK Pflegelotse 2019; eigene Berechnungen nach Schwarze \& Spiekermann 2018 (BBSR

Erreichbarkeitsmodell, Netzmodell DLM 250);

sowie eigene Berechnungen nach Tarifvertrag für den öffentlichen Dienst - Pflege (vom 13. September 2005, letzte Änderung 18. April 2018) und Versorgungsverträge der Träger ambulanter Pflege mit den Pflegekassen (AOK Bayern 2019)

Autoren: Burk, Meyer, Peters Datum: 06.02.2019 
Tabelle 4: Erreichbarkeit nach Landkreisen (Rasterzellen mit Wohnbevölkerung)

\begin{tabular}{|c|c|c|c|c|c|}
\hline $\begin{array}{l}\text { Landkreis/ } \\
\text { kreisfreie Stadt }\end{array}$ & $\begin{array}{l}\text { Anzahl Personen } \\
\text { „80 Jahre und } \\
\text { älter“ 2011* }\end{array}$ & $\begin{array}{l}\text { Anzahl Personen } \\
\text { „80 Jahre und } \\
\text { älter“ } 2017^{\star}\end{array}$ & $\begin{array}{l}\text { Anzahl Personen „80 } \\
\text { Jahre und älter““ } 2025 \\
\text { (vorausberechnet)* }^{*}\end{array}$ & $\begin{array}{l}\text { Maximale } \\
\text { Fahrzeit } \\
\text { (in Minuten) }\end{array}$ & $\begin{array}{l}\text { Durchschnittliche } \\
\text { Fahrzeit } \\
\text { (in Minuten) }\end{array}$ \\
\hline $\begin{array}{l}\text { Aschaffenburg, } \\
\text { kreisfreie Stadt }\end{array}$ & 3.773 & 4.205 & 4.758 & 12,9 & 2,6 \\
\hline $\begin{array}{l}\text { Schweinfurt, } \\
\text { kreisfreie Stadt }\end{array}$ & 3.978 & 4.213 & 4.571 & 9 & 2,9 \\
\hline $\begin{array}{l}\text { Würzburg, } \\
\text { kreisfreie Stadt }\end{array}$ & 7.600 & 8.369 & 9.018 & 12,4 & 3,1 \\
\hline Aschaffenburg & 8.434 & 10.762 & 11.790 & 20,5 & 4,2 \\
\hline Bad Kissingen & 6.851 & 7.657 & 8.284 & 34,5 & 5,5 \\
\hline Rhön-Grabfeld & 4.537 & 5.224 & 5.279 & 30 & 6,4 \\
\hline Haßberge & 4.150 & 4.832 & 4.899 & 24,7 & 6,9 \\
\hline Kitzingen & 4.624 & 5.445 & 5.725 & 18,9 & 4,9 \\
\hline Miltenberg & 6.227 & 7.778 & 8.512 & 31,4 & 6,2 \\
\hline Main-Spessart & 6.979 & 8.395 & 8.998 & 32 & 5,5 \\
\hline Schweinfurt & 5.713 & 7.206 & 7.713 & 19,9 & 5,7 \\
\hline Würzburg & 7.444 & 9.367 & 10.491 & 20,1 & 5,2 \\
\hline
\end{tabular}

* Werte auf ganze Zahlen gerundet. Werte ergeben sich aus Summierungen der Rasterzellen (100x100m) mit wohnhafter Bevölkerung aus dem Zensus 2011, korrigiert durch einen Korrekturfaktor sowie deren Berechnung für 2017 und Vorausberechnung für 2025 (vgl. Kapitel 3.1). Nicht zugeordnete Rasterzellen am Rand des Untersuchungsgebietes: 50 von 55.955.

Quelle: Eigene Berechnung auf Grundlage der Zensusdatenbank der Statistischen Ämter des Bundes und der Länder ${ }^{14}$, der Zensusergebnisse ${ }^{15}$ und auf Anfrage vom Bayerischen Landesamt für Statistik erhaltene Daten (Fortschreibung des Bevölkerungsstandes 2014 bis 2017 sowie Demographie-Spiegel 2014-2028/2034, differenziert nach Altersjahren ab 65 Jahren).

gebedarfs wesentlich beeinflusst wird. Die vorausberechnete Entwicklung für die Jahre 2011 (Zensus) und 2025 ist in Abbildung 7 für die Altersgruppe „80 Jahre und älter" dargestellt und zeigt ein differenziertes Bild. In der überwiegenden Mehrzahl werden die Gemeinden von einem Zuwachs in der entsprechenden Altersgruppe bis zum Jahr 2025 betroffen sein. Lediglich für 73 der 308 Gemeinden Unterfrankens kann von einer rückläufigen oder stagnierenden Entwicklung in dieser Altersgruppe (zwischen $-5 \%$ und $+5 \%$ ) ausgegangen werden. Bei den stärkeren Zuwächsen von mehr als $25 \%$ und mehr als $35 \%$ lässt sich als gewisses Muster die Konzentration von Gemeinden um die kreisfreien Städte Würzburg und Aschaffenburg sowie etwas eingeschränkter auch um Schweinfurt hervorheben

Die Zunahme älterer Menschen in den Umlandgemeinden der Großstädte ist vermutlich eine Folge der

$14 \mathrm{https}: / /$ ergebnisse.zensus2011.de (28.05.2020).

15 https://www.zensus2011.de/DE/Home/Aktuelles/DemografischeGrunddaten.html?nn=3065474 (28.05.2020). starken (Binnen)wanderung von (jungen) Familien in den 1960er- bis 1990er -Jahren dorthin. Insgesamt ist die Zunahme der höheren Altersgruppen, welche für die Untersuchung relevant sind, vor allem durch eine generelle Alterung der Bevölkerung und nur in bestimmten Gemeinden zusätzlich durch rezente Zu- oder Abwanderung zu erklären.

Bei der Auswertung nach Landkreisen (vgl. Tabelle 4) wird noch einmal deutlich, wie dynamisch sich die Entwicklung der Anzahl von Personen in der Altersgruppe „80 Jahre und älter“ in allen Gebietskörperschaften der Kreisebene vollzieht bzw. voraussichtlich vollziehen wird. Eine besonders deutliche Zunahme zwischen $+30 \%$ und $+40 \%$ für 2025 im Vergleich zu 2011 kennzeichnet die Landkreise Würzburg, Aschaffenburg, Miltenberg und Schweinfurt. Vergleichsweise moderatere Entwicklungen hingegen treffen auf die drei kreisfreien Städte Aschaffenburg, Würzburg und Schweinfurt sowie auf die Landkreise Rhön-Grabfeld, Haßberge und Kitzingen zu. Im Hinblick auf die Fahrtzeiten ist vor allem die durchschnittliche Fahrtzeit relevant, um die Erreichbar- 


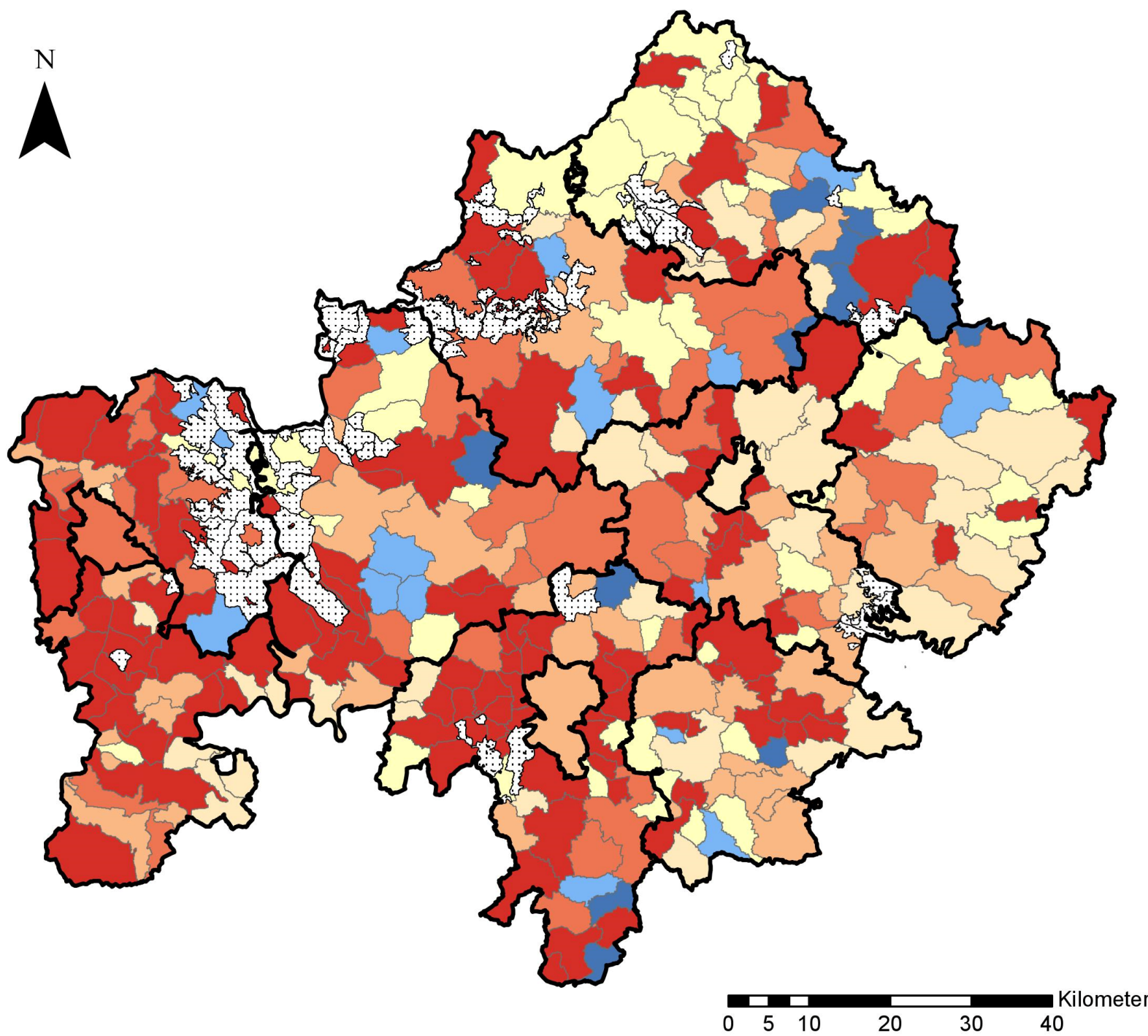

Grenzen Landkreise und Krsfr. Städte

gemeindefreie Gebiete

Vorausberechnete Entwicklung 2011-2025 (\%)

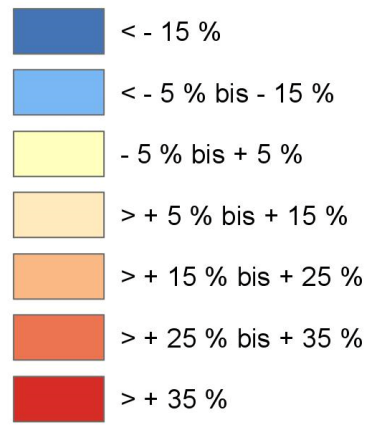

Kartengrundlage: Bayerisches Landesamt für Digitalisierung, Breitband und Vermessung 2019

Quelle: eigene Berechnung auf Grundlage von Statistische Ämter des Bundes und der Länder 2014 (Zensus 2011) sowie Bayerisches Landesamt für Statistik 2018 (Demographie-Spiegel 2014 - 2028/2034)

Autoren: Burk, Meyer, Peters

Datum: 28.01.2019

Abbildung 7: Vorausberechnete Entwicklung der Bevölkerungsgruppe „80 Jahre und älter“ in den Gemeinden Unterfrankens (2011-2025) 
Tabelle 5: Erreichbarkeit, Bevölkerung und Versorgte pro Pflegedienst (Rasterzellen mit Wohnbevölkerung durch Zuordnung zum nächstgelegenen Pflegedienststandort)

\begin{tabular}{|c|c|c|c|c|c|c|}
\hline $\begin{array}{l}\text { Anzahl } \\
\text { Personen „80 } \\
\text { Jahre und } \\
\text { älter“ 2011* } \\
\text { (Mittelwert) }\end{array}$ & $\begin{array}{l}\text { Anzahl } \\
\text { Personen „,80 } \\
\text { Jahre und } \\
\text { älter““ 2017* } \\
\text { (Mittelwert) } \\
\end{array}$ & $\begin{array}{l}\text { Anzahl Personen } \\
\text { „80 Jahre und } \\
\text { älter“ 2025` } \\
\text { (vorausberechnet) } \\
\text { (Mittelwert) }\end{array}$ & $\begin{array}{l}\text { Maximale } \\
\text { Fahrtzeit } \\
\text { (in Minuten) } \\
\text { (Median) }\end{array}$ & $\begin{array}{l}\text { Durchschnittliche } \\
\text { Fahrtzeit (in } \\
\text { Minuten) (Median) }\end{array}$ & $\begin{array}{l}\text { Anzahl } \\
\text { versorgter } \\
\text { Menschen } \\
2017 / 2018^{* *} \\
\text { (Mittelwert) } \\
\end{array}$ & $\begin{array}{l}\text { Geschätzte } \\
\text { Anzahl versorgter } \\
\text { Menschen 2025*** } \\
\text { (Mittelwert) }\end{array}$ \\
\hline 366 & 434 & 469 & 11,9 & 4 & 110 & 117 \\
\hline
\end{tabular}

* Werte auf ganze Zahlen gerundet.

** Werte wurden, sofern vorhanden, aus dem aktuellen Transparenzbericht der ambulanten Pflegedienste entnommen. Werte auf ganze Zellen gerundet.

*** Wert ergibt sich für jeden ambulanten Pflegedienst durch Multiplikation der Anzahl versorgter Menschen (aus dem aktuellen Transparenzbericht des Pflegedienstes) mit dem berechneten Faktor, um den sich die Anzahl der Personen in der Altersgruppe „,80 Jahre und älter“ im modellierten Einzugsbereich des Pflegedienstes zwischen 2017 und 2025 gemäß der Bevölkerungsvorausberechnung verändern wird (Schätzung). Andere Einflussfaktoren wie Kapazitätsgrenzen der Pflegedienste oder fehlendes Personal bei der Aufstockung der Kundenzahl werden hier modellhaft nicht mit einbezogen. Werte auf ganze Zahlen gerundet.

Quelle: Eigene Berechnung auf der Grundlage der Zensusdatenbank der Statistischen Ämter des Bundes und der Länder ${ }^{16}$, der Zensusergebnisse $^{17}$ und auf Anfrage vom Bayerischen Landesamt für Statistik erhaltene Daten (Fortschreibung des Bevölkerungsstandes 2014 bis 2017 sowie Demographie-Spiegel 2014-2028/2034, differenziert nach Altersjahren ab 65 Jahren).

keitsverhältnisse zu charakterisieren. Durch kurze Wege innerhalb des Stadtgebiets sowie eine hohe Dichte an Pflegedienststandorten weisen die drei kreisfreien Städte geringe durchschnittliche Fahrzeiten zwischen 2,6 und 3,1 Minuten auf. Auch im Landkreis Aschaffenburg (4,2 Minuten) oder etwa in den Landkreisen Kitzingen (4,9 Minuten) und Würzburg (5,2 Minuten) finden sich geringe durchschnittliche Fahrzeiten und somit gute durchschnittliche Erreichbarkeiten durch ambulante Pflegedienste. Der höchste Wert findet sich mit durchschnittlich 6,9 Minuten im Landkreis Haßberge.

Die durchgeführte GIS-Modellierung weist jedem Pflegedienststandort in seinem modellierten Einzugsbereich die entsprechenden Rasterzellen mit Wohnbevölkerung samt deren demographischen Informationen und resultierenden Erreichbarkeitsverhältnissen zu. Auch wenn diese Ergebnisse in Form von Tabellen für alle unterfränkischen Pflegedienste vorliegen, werden in diesem Beitrag nur durchschnittliche Kennzahlen, gebildet aus der Gesamtheit aller ambulanten Pflegedienste, ausgewiesen, um an einer übersichtlichen Darstellung festzuhalten und Erfordernisse des Datenschutzes zu wahren.

Tabelle 5 gibt die Bevölkerungsentwicklung, Erreichbarkeitsverhältnisse sowie eine geschätzte Entwicklung der Zahl der Versorgten an, die einen ambulanten

16 https://ergebnisse.zensus2011.de (28.05.2020).

17 https://www.zensus2011.de/DE/Home/Aktuelles/DemografischeGrunddaten.html?nn=3065474 (28.05.2020).
Pflegedienst in Unterfranken in seinem Einzugsbereich durchschnittlich kennzeichnen. Da die Einzugsbereiche der im Modell verwendeten Standorte ambulanter Pflegedienste in einem Radius von 15 Kilometern außerhalb Unterfrankens nicht vollständig abgebildet werden, beziehen sich die nachfolgenden Differenzierungen nur auf Pflegedienste mit Sitz in Unterfranken. Jene einbezogenen Pflegedienste außerhalb Unterfrankens versorgen im Modell insgesamt 1.835 (2011) bzw. 2.318 (2017) Menschen der Altersgruppe „80 Jahre und älter“ in den grenznahen Bereichen Unterfrankens.

Während im Jahr 2011 auf einen ambulanten Pflegedienst in Unterfranken durchschnittlich 366 Personen der Altersgruppe „80 Jahre und älter" kamen, waren es 2017 bereits 434. Gemäß der vorausberechneten Entwicklung werden es im Jahr 2025469 Personen sein. Diese deutliche Steigerung wird in der Berechnung auch ins Verhältnis mit der Zahl der real durch die Pflegedienste versorgten Personen gesetzt. Wie den jeweiligen Transparenzberichten der Jahre 2017 oder 2018 (je nach Datum der letzten Qualitätsprüfung) zu entnehmen ist, versorgt ein ambulanter Pflegedienst in Unterfranken im Schnitt 110 Menschen. Nimmt man die voraussichtliche Veränderungsrate der Altersgruppe „80 Jahre und älter" zwischen 2017 und 2025 an und wendet diese auf die Anzahl der Versorgten, ohne Berücksichtigung anderer potenzieller Einflussfaktoren, an, müsste die Zahl der pro Pflegedienst versorgten Personen bis zum Jahr 2025 also auf 117 ansteigen. Im Gegensatz zu den anderen statistischen Kennzahlen wurde für die Erreich- 
barkeiten der Median statt dem Mittelwert verwendet, um den Einfluss durch Extremwerte (z. B. der maximalen Fahrzeit) zu reduzieren. Für die maximale Fahrzeit werden 11,9 Minuten ausgewiesen. Gerade der Wert für den Median der durchschnittlichen Fahrzeit eines Pflegedienstes in seinem Einzugsbereich befindet sich mit 4,0 Minuten auf einem moderaten Niveau.

\section{Diskussion der Ergebnisse}

Die Ergebnisse der Berechnungen zeigen, dass ein Großteil des Untersuchungsgebietes hinsichtlich der Erreichbarkeiten von ambulanten Pflegediensten relativ gut abgedeckt ist, da der größte Teil der ermittelten Fahrtzeiten von einem ambulanten Pflegedienst zu den zeitlich nächstgelegenen Rasterzellen bei unter 15 Minuten liegt. In Einzelfällen sind außergewöhnlich hohe Fahrtzeiten in peripheren Gebieten des Spessarts oder der Rhön zu verzeichnen. Für diese Einzelfälle ist aufgrund der stark vereinfachten Modellierung eine genauere Einzelbetrachtung notwendig. Sie sollten „bei Interventions- oder Handlungsabsicht (insbesondere auf der Ebene der einzelnen Gemeinde oder Rasterzelle) unter Berücksichtigung realer Gegebenheiten" (Neumeier 2015: 32) verifiziert werden. Vor allem in den kreisfreien Städten ergeben sich aufgrund der hohen Standortdichte sehr gute Erreichbarkeitsverhältnisse. Aufgrund der hohen Bevölkerungsdichten in diesen Gebieten und der aktuellen demographischen Entwicklung sind die Erreichbarkeitsverhältnisse jedoch differenziert von der realen Versorgungssituation zu betrachten.

Ambulante Pflegedienste in ländlichen Gebieten müssen aufgrund der geringeren Standortdichte größere Gebiete versorgen. In einer bundesweiten repräsentativen Befragung "geben ambulante Dienste an, dass eine Versorgung von Klient*innen mit einem Anfahrtsweg von mehr als $25 \mathrm{~km}$ kaum kostendeckend" (DIP 2016: 3) erscheint. Hinsichtlich der in Kapitel 4.1 ermittelten Fahrtzeiten kann jedoch weitestgehend von guten Erreichbarkeitsverhältnissen im Untersuchungsgebiet und kostendeckenden Touren ausgegangen werden.

Die Ergebnisse aus Kapitel 4.2 zeigen, dass die Anfahrtspauschale in den Städten und ihrer unmittelbaren Umgebung sowie entlang von Verkehrsachsen die Kosten für eine flächendeckende Erreichbarkeit garantiert, jedoch besonders in den peripheren Bereichen, wie dem Spessart oder der südlichen Rhön, nicht als geeignetes Instrument zur Deckung der Anfahrtskosten angesehen werden kann, die nach Neumeier (2015: 32) mit $20 \%$ bis $50 \%$ der Betriebskosten ins Gewicht fallen.
Berücksichtigt werden sollten deshalb darüber hinaus weitere fahrzeuggebundene Kosten, wie beispielsweise Benzin, Fahrzeugverschleiß oder Anschaffung.

Somit ist das Modell einer derartigen Pauschale im Allgemeinen zu hinterfragen. Alternativ könnte über eine flexible Pauschale nach dem Vorbild der Entfernungspauschale (auch Pendlerpauschale genannt) diskutiert werden. Bei dieser werden Berufspendlern pro vollendetem Kilometer 0,30 Euro erstattet. ${ }^{18}$ Eine mögliche Abwandlung einer solchen Pauschale könnte eine Erstattung pro vollendeter Minute Fahrzeit sein, die Unterschiede zwischen den höheren Durchschnittsgeschwindigkeiten im ländlichen Raum und den niedrigeren im städtischen Raum (bedingt durch Ampeln und generell höheres Verkehrsaufkommen) nivellieren würde.

Die Anpassung der Anfahrtspauschale ist besonders vor dem Hintergrund des demographischen Wandels zu berücksichtigen. Die Versorgungssicherheit könnte somit zumindest nach dem Kriterium der Erreichbarkeit gewährleistet werden, losgelöst von der Abwägung nach Leistungserbringung vor Ort. Darüber hinaus würde auch die Anpassung der Pflegedienste an die aktuellen Trends verbessert werden. Bei einer zunehmenden Zahl Pflegebedürftiger bei gleichzeitigem zu erwartendem steigendem Konkurrenzdruck um Fachkräfte wären diese flexibler bei Zentralisierungen mit Bündelung von Kräften bei gleichzeitiger Ausweitung der Einzugsgebiete.

Das Fehlen einer standardisierten Tourenplanung in der Praxis ist aus ,theoretischer' Sicht durchaus kritisch zu betrachten, da hierdurch eine genaue Kalkulation von Versorgungsgrenzen und somit Prognosen über eine Veränderung in der Versorgungslage erheblich erschwert werden. In der Praxis ermöglicht die fehlende Standardisierung ohne klare Definition von Grenzen der rentablen Fahrtzeit bzw. Fahrtkosten jedoch auch eine entsprechende Flexibilität in der Tourenplanung. Es muss außerdem erwähnt werden, dass neben betriebswirtschaftlichen Interessen der Wohlfahrtsgedanke, in diesem Fall die Versorgung aller Pflegebedürftigen ungeachtet vom Standort und in Abstimmung mit anderen ambulanten Pflegediensten, eine zentrale Rolle bei den meisten Pflegediensten, insbesondere mit freigemeinnützigen Trägern, einnimmt (Interview 3 2018: 2).

Die Einbeziehung demographischer Informationen in die GIS-Modellierung hat gezeigt, dass die ambulante

18 Gesetz zur Änderung und Vereinfachung der Unternehmensbesteuerung und des steuerlichen Reisekostenrechts vom 20. Februar 2013. 
Pflegeinfrastruktur in Unterfranken durchaus vor großen Herausforderungen steht, die durch den Zuwachs der Gruppe älterer, potenziell pflegebedürftiger Menschen bedingt sind. Auch komplexe Untersuchungen, welche mit Szenarien und konkreten Werten der Schätzung von Pflegebedürftigen argumentieren, wie beispielsweise im Rahmen der Pflegebedarfsplanung des seniorenpolitischen Gesamtkonzepts von Stadt und Landkreis Würzburg (vgl. ARGE 2016: 58), gehen von kontinuierlichen Zuwächsen der Nachfrage nach (ambulanten) Pflegeleistungen aus. Auch wenn diese Untersuchungen sehr differenziert die Entwicklung der regionalen Nachfragestrukturen erfassen, so sind für Unterfranken keine umfassenden Gegenüberstellungen der aktuellen sowie zukünftig zu erwartenden Entwicklung von Pflegediensten und Pflegepersonal bekannt. Hierfür können an dieser Stelle nur Aussagen für den regionsübergreifenden bundesweiten Kontext getroffen werden.

Nach Angaben der Bundesagentur für Arbeit (2018) besteht in der Altenpflege ein flächendeckender, bundesweiter Fachkräftemangel. Dieser bezieht sich spezifisch auf examinierte Pflegefachkräfte. Bei Altenpflegehelfern besteht hingegen kein Engpass. Hier überschreitet, bundesweit betrachtet, das Angebot die Nachfrage an Pflegehelfern. Der ,doppelte demographische Wandel', der gleichzeitig für einen Rückgang des Angebotspotenzials an Pflegekräften sowie für eine steigende Nachfrage nach Pflegedienstleistungen verantwortlich ist, könnte Schätzungen zufolge dazu führen, dass im Jahr 2030 mehr als jede vierte Stelle in der Altenpflege nicht besetzt werden kann, wie eine Studie im Auftrag des Bundesministeriums für Wirtschaft und Energie zeigt (Neldner/ Hofmann/Peters et al. 2017: 100).

In den geführten Interviews wurden ähnliche Problematiken für den Untersuchungsraum Unterfranken thematisiert. Auch hier stellt der Fachkräftemangel eine Herausforderung dar, da ambulante Pflegedienste zunehmend Probleme haben, qualifiziertes Personal zu finden. Dies führt nach Aussagen von Interviewpartnerinnen und -partnern auch bereits dazu, dass potenzielle Pflegekunden vereinzelt aufgrund erreichter Kapazitätsgrenzen bei Anfragen abgewiesen bzw. auf Wartelisten gesetzt werden müssen (Interview 3 2018: 1; Interview 5 2019: 2). Da sich die Geschäftsmodelle und Arbeitsweisen von ambulanten Pflegediensten zum Teil deutlich unterscheiden, ist es kaum möglich, erfolgreiche übergreifende Strategien zur Fachkräftesicherung zu identifizieren. Gleichzeitig ist die Thematik in den vergangenen Jahren auch auf landes- und bundespolitischer Ebene zunehmend aufgetreten. Die Bundesregierung strebt daher beispielsweise mit dem 2017 verabschiedeten
Gesetz zur Reform der Pflegeberufe ${ }^{19}$ an, die Pflegeausbildung attraktiver zu gestalten und durch eine Anpassung der Ausbildungsstruktur flexiblere Einsatzmöglichkeiten für Pflegekräfte zu schaffen. Angesichts der auch in dieser Untersuchung für Unterfranken herausgestellten demographischen Entwicklungen und den resultierenden Herausforderungen ist es notwendig, eine Verbesserung der Angebotsstruktur in der ambulanten Pflege zu erreichen, um langfristig eine ausreichende, flächendeckende Versorgung zu gewährleisten.

\section{Methodenkritik}

Die Recherche der Standorte ambulanter Pflegedienste wurde mittels zweier Suchmaschinen (vgl. Kapitel 3.3) durchgeführt. Die recherchierten Listen der Pflegedienste im Untersuchungsgebiet sollten somit zwar den weitaus größten Teil aller zugelassenen ambulanten Pflegedienste abdecken, sie beziehen sich aber stets auf den Zeitpunkt der Erhebung mit Stand zum Ende des Jahres 2018. Des Weiteren konnten für die Pflegedienste die Leistungsspektren nicht immer eindeutig identifiziert werden. Die Einordnung wurde für jeden Einzelfall auf der Basis der vorhandenen Transparenzberichte Pflege oder des Online-Auftritts der Pflegedienste durchgeführt.

Die Ergebnisse der rasterbasierten Erreichbarkeitsanalyse hängen in starkem Maße von den Daten, die der Berechnung zugrunde liegen, ab (vgl. Neumeier 2015: 34). Die Erreichbarkeitsanalyse der ambulanten Pflegedienste modelliert weniger reale Entscheidungsprozesse, da „weder Kundenentscheidungen im Hinblick auf die Wahl des Pflegedienstes noch Differenzen im Angebots-/Leistungsspektrum, Wegeketten (d.h. Verknüpfung mehrerer Einsatzorte im Rahmen der Tagestour), Betreuungskapazitäten auf Seiten der Pflegedienste oder reale Einzugsbereiche einzelner Ambulanter Pflegedienste [...] berücksichtigt [werden]" (Neumeier 2015: 31). Vielmehr sollen mit den Ergebnissen des Modells generelle Aussagen zur grundlegenden Verfügbarkeit und Erreichbarkeitssituation im Untersuchungsgebiet getroffen werden können, welche dennoch fundierte und hoch aufgelöste Informationen zur Versorgung durch ambulante Pflegedienste liefern.

Im Rahmen der vektoriellen Modellierung konnte die in der Praxis gebräuchliche kombinierte Tourenplanung ebenfalls nicht berücksichtigt werden, da in den Interviews deutlich wurde, dass es hierfür keine standardisier-

19 Pflegeberufegesetz vom 17. Juli 2017, das zuletzt durch Artikel 9 des Gesetzes vom 19. Mai 2020 geändert worden ist. 
ten Vorgaben gibt. Eine Tour ist einzig durch die Dauer begrenzt (sechs Stunden, danach Anspruch auf Pause), nicht jedoch durch Distanzen. Das führt dazu, dass die hier ermittelte maximale Reichweite in der Praxis überschritten werden kann. Eine kombinierte Tour, die eine lange Anfahrt hat, könnte sich rentieren, sofern im jeweiligen Zielgebiet genug Leistungen erbracht werden, um die Anfahrt zu refinanzieren. Des Weiteren ist zu berücksichtigen, dass, wie bereits in Kapitel 3.3.4 erwähnt, nur die Personalkosten zur Berechnung herangezogen werden. Diese machen jedoch auch $50 \%$ bis $80 \%$ der Gesamtkosten eines ambulanten Pflegedienstes aus und liegen im besten Fall bei $70 \%$ bis $75 \%$ (vgl. PPM 2016). ${ }^{20}$ Eine Kalkulation von Sprit-, Verschleißund Anschaffungskosten war aufgrund der Wahl von Zeitgrenzen im Modell technisch nicht möglich. Nichtsdestotrotz eignet sich die verwendete Methodik zur Diskussion der Höhe und Sinnhaftigkeit der derzeitigen Anfahrtspauschale, da die dargestellten Fahrtzeitzonen potenzielle Einzugsgebiete für ambulante Pflegedienste aufzeigen und somit Rückschlüsse auf etwaige Versorgungslücken zulassen.

\section{Fazit und Ausblick}

Aus den Ergebnissen unserer Untersuchung gehen für den Regierungsbezirk Unterfranken flächendeckend relativ gute Erreichbarkeitsverhältnisse hervor. Es ist jedoch nicht möglich, aus den Ergebnissen der Erreichbarkeitsanalysen unmittelbar auf die aktuelle Versorgungssituation zu schließen, da weitere Faktoren wie das Angebots- und Leistungsspektrum der Dienste, der Personalmangel oder die steigende Anzahl der Pflegebedürftigen berücksichtigt werden müssen. Trotz der Restriktionen des Modells bringt die gewählte Methode belastbare Ergebnisse hervor, da sie sich auf den Faktor Zeit als Impedanzvariable, der vor allem in ländlichen Räumen ein wichtiger Kostenfaktor für ambulante Pflegedienste darstellt, stützt. Aus den Experteninterviews ging hervor, dass Pflegedienste schon jetzt potenzielle Kunden abweisen müssen, da Kapazitätsgrenzen erreicht und Versorgungslücken sowohl in städtischen als auch ländlichen Gebieten auszumachen sind. Diese Aufnahmestopps resultieren meist aus Personalmangel, obgleich manche Pflegedienste ihre Touren bereits ausschließlich mit Pflegehelfern durchführen und dabei auch auf angeworbene ausländische Pflegekräfte zurückgrei-

20 https://www.ppm-online.org/blog/kennzahl-anteil-lohnkostenan-gesamtkosten/ (28.05.2020). fen (Interview 2 2018: 3 ff.; Interview 5 2019: 1 f.) Gerade im Hinblick auf die demographische Entwicklung ist davon auszugehen, dass sich der Pflegebedarf erhöhen wird und dieser bei aktueller Versorgungslage nicht gedeckt werden kann.

In den kreisfreien Städten des Regierungsbezirkes ist zwar eine hohe Dichte ambulanter Pflegedienste zu verzeichnen, allerdings sind diese Teilräume gleichzeitig auch am dichtesten besiedelt. In den umliegenden Gemeinden der kreisfreien Städte ist mit einem überproportionalen Anstieg der für diese Studie relevanten Bevölkerungsgruppe der über 80-Jährigen zu rechnen. Es ist jedoch fraglich, ob die städtischen Pflegedienste die Versorgung des Umlandes hinsichtlich der steigenden Nachfrage und bestehender Personalengpässe tragen können. Zudem besteht aufgrund der hohen Nachfrage keine (wirtschaftliche) Notwendigkeit seitens der Pflegedienste, das Umland mit zu versorgen, und es ist für die städtischen Pflegedienste aufgrund der erstatteten Anfahrtspauschale deutlich rentabler, ihre Touren auf kleinere Gebiete zu begrenzen und stadtteilbezogene Touren zu planen. Des Weiteren ist es von Seite der Pflegepatienten oftmals gewünscht, den Ansprechpartner, also die Geschäftsstelle des Pflegedienstes, in unmittelbarer Nähe zu haben (Interview 5 2019: 3).

Aus den Ergebnissen der Erreichbarkeitsanalysen ergeben sich auch Gebiete schlechterer Erreichbarkeit. Diese sind vor allem in Regionen mit schlechter verkehrlicher Anbindung auszumachen. Aus den Ergebnissen des vektoriellen Ansatzes bleibt das Modell der Anfahrtspauschale zu hinterfragen. Womöglich bedarf es langfristig gesehen neuer Modelle der Fahrtkostenerstattung, um die Versorgungssicherheit in peripheren Regionen zu gewährleisten. Insgesamt liegt die Herausforderung der Versorgungssicherung jedoch nicht nur bei der gesamträumlichen Erreichbarkeit der ambulanten Pflegedienste, sondern ergibt sich auch aus den Kapazitätsgrenzen der einzelnen Pflegedienste.

Mögliche Lösungsansätze in Bezug auf die Erreichbarkeit ambulanter Pflegedienste könnten sich durch die Eröffnung von Zweigstellen städtischer Pflegedienste in den Bereichen um die kreisfreien Städte ergeben. Von diesen Zweigstellen könnten außerstädtische Touren gefahren werden, welche aufgrund der dispersen Siedlungsstruktur und weitreichenderen Versorgungsbereichen jedoch auf eine alternative Fahrtkostenrefinanzierung angewiesen wären. Auch derAnsatz der dezentralen Versorgung durch Pflegekräfte vom eigenen Wohnort mittels Privat-Pkw könnte ein zukunftsfähiges Modell sein. Hier gibt es bereits etablierte Geschäftsmodelle. Außerdem ermöglichen immer mehr Pflegedienste ihren 
Mitarbeiterinnen und Mitarbeitern, die betriebseigenen Einsatzfahrzeuge nach der Tour am Wohnort abzustellen und die bevorstehenden Touren vom Wohnort aus zu steuern, um Fahrtzeiten und Fahrtkosten zu senken (Interview 2 2018: 11; Interview 4 2019: 3 f.).

Inwiefern die ambulanten Pflegedienste im „Pflegemix aus professionellen Leistungserbringern, Familienangehörigen und [...] ehrenamtlichen Helfern" (Niens/ Marggraf/Hoffmeister 2015: 32) beitragen können, die aktuellen und künftigen Herausforderungen zu bewältigen und die Versorgungssicherheit zu gewährleisten, hängt stark von deren Betreuungskapazitäten ab, welche sich nicht zuletzt aus dem verfügbaren Pflegepersonal ergeben. Pflegeberufe stehen aktuell mehr denn je in regem politischen und gesellschaftlichen Diskurs. Eine Attraktivitätssteigerung pflegerischer Berufe ist demnach notwendig, um die heimische Versorgung durch professionelle Pflegedienstleister bedarfsgerecht sicherstellen zu können.

\section{Literatur}

Ahlmeyer, F.; Wittowsky, D. (2018): Was brauchen wir in ländlichen Räumen? Erreichbarkeitsmodellierung als strategischer Ansatz der regionalen Standort- und Verkehrsplanung. In: Raumforschung und Raumordnung | Spatial Research and Planning 76, 6, 531-550. doi: 10.1007/s13147-018-0558-8

ARGE - Arbeitsgemeinschaft Sozialplanung in Bayern (2016): Fortschreibung des Seniorenpolitischen Gesamtkonzepts für den Landkreis Würzburg. Augsburg/München.

Bundesagentur für Arbeit (2018): Arbeitsmarktsituation im Pflegebereich. Nürnberg.

Dahlgren, A. (2008): Geographic Accessibility Analysis. Methods and Application. Dissertation am Lund Institute of Technology.

DIP - Deutsches Institut für angewandte Pflegeforschung (2016): Pflege-Thermometer 2016. Eine bundesweite Befragung von Leitungskräften zur Situation der Pflege und Patientenversorgung in der ambulanten Pflege. Köln.

Eckey, H.-F.; Kosfeld, R.; Türck, M. (2008): Deskriptive Statistik. Wiesbaden.

Heit, F.; Köppe, P.; Kratschke, M.; Reichenwallner, S. (2014): Herausforderungen für die Gesundheitsversorgung ländlicher Räume vor dem Hintergrund des demographischen Wandels. Das Fallbeispiel der durch Verwaltungsgrenzen zerschnittenen Fränkischen Schweiz. In: Mitteilungen der Fränkischen Geographischen Gesellschaft 59, 131-147.

Mittelstaedt, E. (1998): Abgrenzung von Grund- und Behandlungspflege aus sozialrechtlicher und ökonomischer Perspektive. In: Pflege und Gesellschaft 3, 2, 7-10.

Neldner, T.; Hofmann, E.; Peters, V.; Richter, T.; Hofmann, S.; Hans, J. P.; Stohr, D.; Koch, A.; Späth, J. (2017): Entwicklung der Angebotsstruktur, der Beschäftigung sowie des Fachkräftebedarfs im nichtärztlichen Bereich der Gesundheitswirtschaft.
Studie im Auftrag des Bundesministeriums für Wirtschaft und Energie. Endbericht. Berlin/Darmstadt/Tübingen.

Neumeier, S. (2014): Modellierung der Erreichbarkeit von Supermärkten und Discountern. Untersuchung zum regionalen Versorgungsgrad mit Dienstleistungen der Grundversorgung. Braunschweig. $=$ Thünen Working Paper 16.

Neumeier, S. (2015): Lokale Verteilung Ambulanter Pflegedienste nach SGB XI in Deutschland auf Basis eines rasterbasierten GIS-Erreichbarkeitsmodells. Braunschweig. = Thünen Working Paper 47.

Neumeier, S. (2016): Regional Distribution of Ambulant Nursing Services in Germany. A GIS Accessibility Analysis. In: Raumforschung und Raumordnung 74, 4, 339-359. doi: 10.1007/ s13147-016-0409-4

Niens, C.; Marggraf, R.; Hoffmeister, F. (2015): Ambulante Pflege im ländlichen Raum. Überlegungen zur effizienten Sicherstellung von Bedarfsgerechtigkeit. Göttingen. = Diskussionspapiere des Departments für Agrarökonomie und rurale Entwicklung der Universität Göttingen 1513.

Rauch, S.; Rauh, J. (2016): Verfahren der GIS-Modellierung von Erreichbarkeiten für Schlaganfallversorgungszentren. In: Raumforschung und Raumordnung 74, 5, 437-450. doi: 10.1007/s13147-016-0432-5

Rauh, J.; Rauch, S. (2019): Konzeptionelle Überlegungen zu fußläufigen GIS-gestützten Erreichbarkeitsanalysen in der Nahversorgung in ländlichen Räumen. In: Neiberger, C.; Pez, P. (Hrsg.): Einzelhandel und Stadtverkehr. Neue Entwicklungstendenzen durch Digitalisierung und Stadtgestaltung. Würzburg, 37-49. = Geographische Handelsforschung 28. doi: 10.25972/WUP-978-3-95826-113

Schwarze, B.; Spiekermann, K. (2019): Methodische Weiterentwicklungen der Erreichbarkeitsanalysen des BBSR. Bonn. = BBSR-Online-Publikation 09/2019.

Schwinger, A.; Tsiasioti, C. (2018): Pflegebedürftigkeit in Deutschland. In: Jacobs, K.; Kuhlmey, A.; Greß, S.; Klauber, J.; Schwinger, A. (Hrsg.): Pflege-Report 2018. Qualität in der Pflege. Berlin, 173-204. doi: 10.1007/978-3-662-56822-4_16

Spiekermann, K.; Wegener, M. (2012): Dimensionen der Erreichbarkeit - von global bis lokal. In: RaumPlanung 165, 6, 8-14.

Statistisches Bundesamt (2018): Pflegestatistik. Pflege im Rahmen der Pflegeversicherung. Deutschlandergebnisse 2017. Wiesbaden. 